\title{
OSCILLATION AND NONOSCILLATION OF SOLUTIONS OF GENERALIZED EMDEN-FOWLER EQUATIONS
}

BY

\author{
C. V. COFFMAN( $\left.{ }^{1}\right)$ AND J. S. W. WONG
}

\begin{abstract}
This paper treats the ordinary differential equation $y^{\prime \prime}+y F\left(y^{2}, x\right)=0$, $x>0$, where $y F\left(y^{2}, x\right)$ is continuous in $(y, x)$ for $x>0,|y|<\infty$, and $F(t, x)$ is nonnegative; the equation is assumed to be either of sublinear or superlinear type. Criteria are given for the equation to be oscillatory, to be nonoscillatory, to possess oscillatory solutions or to possess nonoscillatory solutions. An attempt has been made to unify the methods of treatment of the sublinear and superlinear cases. These methods consist primarily of comparison with linear equations and the use of "energy" functions. An Appendix treats the questions of continuability and uniqueness of solutions of the equation considered in the main text.
\end{abstract}

1. Introduction. We are here concerned with the oscillatory behavior of solutions of second order nonlinear ordinary differential equations of the form

$$
y^{\prime \prime}+y F\left(y^{2}, x\right)=0, \quad x>0,
$$

where $y F\left(y^{2}, x\right)$ is continuous for $x>0$ and $|y|<\infty$, and $F(t, x)$ is nonnegative for $x>0$ and $t>0$. The prototype of equation (1) is the following:

$$
y^{\prime \prime}+q(x)|y|^{\gamma} \operatorname{sgn} y=0, \quad x>0,
$$

where $q(x) \geqq 0$ and $\gamma>0$. Both equations (1) and (2) include the so called "EmdenFowler equation":

$$
y^{\prime \prime}+x^{\sigma}|y|^{\gamma} \operatorname{sgn} y=0, \quad x>0,
$$

where $\gamma>0$ and $\sigma$ is real. In the discussion to follow, it is convenient to classify equation (1) according to the nonlinearity of $F$, namely (1) is in the superlinear case if $F(t, x)$ is monotone increasing in $t$ for every $x$ and similarly it is in the sublinear case if $F(t, x)$ is monotone decreasing in $t$ for every $x$. In $\S 2$, we also introduce weaker notions of super and sublinearity, which play an important role in the oscillation and nonoscillation of solutions of equation (1).

A nontrivial solution of (1) is said to be nonoscillatory if, for every $a>0$, the

Received by the editors March 16, 1970.

AMS 1969 subject classifications. Primary 3442, 3445.

Key words and phrases. Oscillation, nonoscillation, nonlinear.

( $\left.{ }^{1}\right)$ The research of the first-named author was in part supported by NSF GP-7662, and was in part performed at the U.S.A.F. Aerospace Research Laboratories while the author was there in the capacity of an Ohio State University Research Foundation Visiting Research Associate under Contract F33615-67-C-1758. 
number of its zeros in $[a, \infty)$ is finite, and it is said to be oscillatory otherwise. Unlike the linear equation, where the function $F(t, x)$ is independent of $t$, the nonlinear equation may possess solutions of either kind. In view of this, one is led to consider the following types of oscillation and nonoscillation conditions, namely, those which guarantee that all solutions of (1) oscillate and its converse, i.e. the existence of one nonoscillatory solution, and those which guarantee that all solutions of (1) do not oscillate and its converse, i.e. the existence of one oscillatory solution. The first type of oscillation and nonoscillation conditions have been the centre of a considerable amount of research and there are a number of results in this direction for equation (1) or similar equations. We refer the reader to Wong [15] for an expository account of this subject and for other related references. Results of the second type may also be found in Atkinson [1], Wong [14], Macki and Wong [10], Heidel [6] and others. However, in contrast to results of the first type, these results are not sharp when applied to the Emden-Fowler equation. Sharp results have been found recently for equation (2) when $\gamma>1$ by Jasný [7] and Kurzweil [9] for the existence of one oscillatory solution and by Kiguradze [8] and Nehari [13] for nonoscillation. In the sublinear case when $\gamma<1$, Belohorec [2] has obtained results in both directions. For the more general equation (1), in the superlinear case, a study was initiated in Nehari [13] and continued in Coffman and Wong [3], but as far as we know the corresponding sublinear case has not been investigated.

In the present work, we attempt to present a unified treatment for the study of this specific oscillation problem of second type both in the sublinear and the superlinear case. At the same time, we initiate a systematic investigation in the use of Lyapunov-like functions to study oscillation and nonoscillation problems concerning second order nonlinear equations. In the Appendix, we further this approach by showing how it may be used in the study of the continuability problem and the uniqueness of the zero solution. This technique was first introduced by Coffman and Wong [3] for a special case of equation (1) and was suggested by some ingenious differential identities and inequalities used by Nehari in [13]. The main results, too detailed to describe here, include oscillation and nonoscillation theorems for both of the two classes of equation (1) and contain as special cases all of the results cited above. In fact, in the process of this generalization, we not only achieve a certain degree of simplification but also discover improved versions of earlier results even in the simple case of equation (2). For example, Corollary 2 and Corollary 5 improve the results of Belohorec [2] for equation (2) when $\gamma<1$, Corollary 10 refines the result by Jasný [7] and Kurzweil [9] for equation (2) when $\gamma>1$, and Proposition (*) extends a well-known result of Nehari for equation (1) in the superlinear case.

In most of the analysis concerning oscillation of solutions of nonlinear equations, it is often assumed that every locally defined solution of (1) is continuously extendable throughout the entire nonnegative real axis. We have included in this 
paper an Appendix which we hope presents an up-to-date discussion of the continuability problem as well as the problem of local uniqueness of the zero solution. The results presented in the Appendix are used throughout this paper and seem to be of independent interest in themselves.

Finally, we remark that preliminary versions of some of the results given below were announced earlier in [4].

2. Preliminaries. In this section we introduce some notation, formalize usage of certain terminology, and discuss general properties of equation (1).

We assume here that $F(t, x)$ is continuous and nonnegative for $t, x>0$, and that $y F\left(y^{2}, x\right)$ can be defined as a continuous function for $x>0,|y|<\infty$. These conditions suffice for local existence of solutions of the initial value problem

$$
y\left(x_{1}\right)=a, \quad y^{\prime}\left(x_{1}\right)=b, \quad x_{1}>0,
$$

for (1).

A solution of (1) is understood to be a $C^{2}$ function satisfying (1) and defined on a right maximal interval of existence. The term "local solution" will be used to refer to a solution not necessarily defined on a right maximal interval of existence. The term "oscillatory" will be used in the sense of oscillatory on the right, i.e. a solution of (1) is oscillatory if its zeros have a right cluster point, and nonoscillatory otherwise. A cluster point of zeros of a solution of (1) can occur in the interior of its interval of definition, thus a nonoscillatory solution may possess a left continuation which is oscillatory. Finally we remark that any solution with a bounded right maximal interval of existence is necessarily oscillatory. (Cf. Lemma A1 in the Appendix.)

Concerning the classification of equation (1) as superlinear or sublinear, (1) should, strictly speaking, be called superlinear only if $F$ satisfies

$$
F\left(t_{2}, x\right) \geqq F\left(t_{1}, x\right), \quad t_{2}>t_{1}, \quad x \in(0, \infty),
$$

and sublinear only if $F$ satisfies

$$
F\left(t_{2}, x\right) \leqq F\left(t_{1}, x\right), \quad t_{2}>t_{1}, \quad x \in(0, \infty) .
$$

Here we use these terms somewhat more loosely and refer to (1) as superlinear or sublinear respectively if the coefficient satisfies some weaker condition than (4) or (5) under which the typical oscillation or nonoscillation properties of the strictly sublinear or superlinear equation are preserved. Such a condition of generalized superlinearity is

$$
G(t, x) \leqq K t F(t, x), \quad K>0, \quad 0 \leqq t<\infty, \quad 0<x<\infty
$$

the analogous condition of generalized sublinearity is

$$
G(t, x) \geqq K t F(t, x), \quad K>0, \quad 0 \leqq t<\infty, \quad 0<x<\infty .
$$

Here

$$
G(t, x)=\int_{0}^{t} F(s, x) d s=2 \int_{0}^{\sqrt{ } t} s F\left(s^{2}, x\right) d s .
$$


(The assumptions made above concerning $F(t, x)$ clearly imply that $G(t, x)$ is well defined.) Another condition of superlinearity, intermediate between (4) and (6), is

$$
F\left(t_{2}, x\right) \geqq c F\left(t_{1}, x\right), \quad c>0, \quad t_{2}>t_{1}, \quad 0<x<\infty .
$$

Reversing the inequality in (9), we obtain a condition of sublinearity intermediate between (5) and (7), namely,

$$
F\left(t_{2}, x\right) \leqq c F\left(t_{1}, x\right), \quad c>0, \quad t_{2}>t_{1}, \quad 0<x<\infty .
$$

We note that (4) and (5) both are satisfied by the linear equation (1), and the overlap of (6) and (7) is even greater. In fact (6) and (7) are both satisfied by (2) for all $\gamma>0$. Proper sublinearity, for example, which rules out even the linear equation, is obtained by simultaneously requiring (7), (23) and (30).

A further word may be appropriate here concerning the pathologies which solutions of (1) can exhibit. The two major problems are those of global existence and of uniqueness. The former problem arises primarily in the superlinear case, the latter primarily in the sublinear case. In fact, as we shall prove in the Appendix, in the sublinear case, under assumption (5) or under the weaker assumption (10), all solutions of (1) will have an unbounded right maximal interval of existence. This is probably not true if we assume only (7) and is certainly false in the superlinear case, as is well known.

Nonuniqueness can arise due to the fact that we have not assumed a Lipschitz condition on $F$. For the superlinear case this difficulty could easily be eliminated by imposing a locally uniform Lipschitz condition in $t$, however, in the sublinear case, in order to avoid ruling out the equation (2) (with $0<\gamma<1$ ) the most that we can assume is that $F(t, x)$ satisfies a local Lipschitz condition in $t$ for all $x$ but only for $t>0$. In the presence of this latter assumption we will still be confronted with the possibility of nonuniqueness of solutions of the initial value problem (3) for (1) when $a=0$. A more detailed analysis of these problems will be given in the Appendix.

An important technique in the analysis to follow is the rather simple one of comparing equation (1), "along a solution", with a nonoscillatory linear equation

$$
z^{\prime \prime}+p(x) z=0
$$

or with an oscillatory linear equation

$$
u^{\prime \prime}+r(x) u=0
$$

where $p(x)$ and $r(x)$ are positive and continuous on $(0, \infty)$. The following results are obtained as elementary consequences of the Sturm comparison theorem. We assume below that equation (12) is oscillatory and that equation (11) is nonoscillatory. 
Lemma 1. Let $y(x)$ be a nonoscillatory solution of (1). Then

$$
\liminf _{x \rightarrow \infty}(r(x))^{-1} F\left(y^{2}(x), x\right) \leqq 1,
$$

and in fact, there exists an infinite sequence $\left\{\xi_{n}\right\}$ with $\xi_{n} \rightarrow \infty$ as $n \rightarrow \infty$ such that for all $n=1,2, \ldots$

$$
\left(r\left(\xi_{n}\right)\right)^{-1} F\left(y^{2}\left(\xi_{n}\right), \xi_{n}\right) \leqq 1 .
$$

LEMMA 2. Let $y(x)$ be a solution of (1). If for some $x_{0} \geqq 0$,

$$
F\left(y^{2}(x), x\right) \leqq p(x), \quad x_{0} \leqq x<\Omega,
$$

then $y(x)$ is nonoscillatory.

LEMMA 3. Let $y(x)$ be a solution of (1). If for some $x_{0}>0$,

$$
F\left(y^{2}(x), x\right) \geqq r(x), \quad x_{0} \leqq x<\Omega,
$$

then $y(x)$ is oscillatory.

In Lemmas 2 and $3, \Omega=\Omega(y)$ denotes the right endpoint of the right maximal interval of existence of the solution $y(x)$ of (1).

Even more basic to this work than the comparison technique described above is the use of certain Lyapunov type energy functions which are defined for solutions of (1) in terms of certain sufficiently smooth auxiliary functions $\omega$ and $\psi$. Let $y(x)$ be a solution of (1); we define $\Sigma_{x_{0}}(x)=\Sigma_{x_{0}}(x, y(x))$ by

$$
\begin{aligned}
\Sigma_{x_{0}}(x)= & \omega^{2}\left(\psi y^{\prime}-\psi^{\prime} y\right)^{2}(x)-\int_{x_{0}}^{x}\left(\psi y^{\prime}-\psi^{\prime} y\right)^{2}\left(\omega^{2}\right)^{\prime} d x \\
& +\omega^{2} \psi \psi^{\prime \prime} y^{2}(x)+\omega^{2} \psi^{2} G\left(y^{2}, x\right) .
\end{aligned}
$$

For $y$ as above, and for a fixed choice of $x_{0}, \omega$ and $\psi$, we will refer to $\Sigma_{x_{0}}(x)$ as "the energy function for $y$ ". We remark that the "natural" energy function for a solution of (1) is obtained by taking $\omega(x) \equiv \psi(x) \equiv 1$. The introduction of the auxiliary functions $\omega$ and $\psi$ serves in place of a change of dependent and independent variable in equation (1).

Lemma 4. Let $\omega$ and $\psi$ be positive on $(0, \infty)$ with $\omega \in C^{2}(0, \infty)$, and $\psi \in C^{3}(0, \infty)$ and let $y$ be a solution of (1). (i) If $\omega^{2}(x) \psi^{2}(x) G\left(\alpha \psi^{2}(x), x\right)$ is a nondecreasing function of $x$ for every $\alpha>0$, then

$$
\Sigma_{x_{0}}\left(x_{2}\right)-\Sigma_{x_{0}}\left(x_{1}\right) \geqq \frac{1}{2} \int_{x_{1}}^{x_{2}}\left[\omega^{2}\left(\psi^{2}\right)^{m}+\left(\omega^{2}\right)^{\prime} \psi \psi^{\prime \prime}\right] y^{2} d x, \quad x_{2} \geqq x_{1} .
$$

(ii) If $\omega^{2}(x) \psi^{2}(x) G\left(\alpha \psi^{2}(x), x\right)$ is a nonincreasing function of $x$ for every $\alpha>0$, then

$$
\Sigma_{x_{0}}\left(x_{2}\right)-\Sigma_{x_{0}}\left(x_{1}\right) \leqq \frac{1}{2} \int_{x_{1}}^{x_{2}}\left[\omega^{2}\left(\psi^{2}\right)^{m}+\left(\omega^{2}\right)^{\prime} \psi \psi^{\prime \prime}\right] y^{2} d x, \quad x_{2} \geqq x_{1}
$$


Proof. We assume first that $F$ is of class $C^{1}$. Let $y$ be any $C^{2}$ function (not necessarily a solution of (1)), then if $\Sigma_{x_{0}}(x)$ is defined by (13), $\Sigma_{x_{0}}^{\prime}(x)$ can be computed as follows:

$$
\begin{aligned}
\Sigma_{x_{0}}^{\prime}(x)= & \omega^{2}\left[\left(\psi y^{\prime}-\psi^{\prime} y\right)^{2}\right]^{\prime}+\left(\omega^{2} \psi \psi^{\prime \prime} y^{2}\right)^{\prime} \\
& +\left(y^{2} / \psi^{2}\right)^{\prime} \omega^{2} \psi^{2} \partial G\left(\psi^{2} \sigma, x\right) /\left.\partial \sigma\right|_{\sigma=(y / \psi)^{2}}+Q\left(y^{2}, x\right)
\end{aligned}
$$

where

$$
Q\left(y^{2}, x\right)=\left.(\partial / \partial \sigma)\left(\omega^{2}(\sigma) \psi^{2}(\sigma) G\left(\psi^{2}(\sigma)(y(x) / \psi(x))^{2}, \sigma\right)\right)\right|_{\sigma=x} .
$$

Using (8) and rearranging terms, we obtain

$$
\Sigma_{x_{0}}^{\prime}(x)=2 \omega^{2} \psi\left(\psi y^{\prime}-\psi^{\prime} y\right)\left(y^{\prime \prime}+y F\left(y^{2}, x\right)\right)+\frac{1}{2}\left(\omega^{2}\left(\psi^{2}\right)^{m}+\left(\omega^{2}\right)^{\prime} \psi \psi^{\prime \prime}\right) y^{2}+Q\left(y^{2}, x\right) .
$$

Suppose now that condition (i) holds, then $Q\left(y^{2}, x\right) \geqq 0$, and thus an integration of (17) yields

$$
\begin{aligned}
\Sigma_{x_{0}}\left(x_{2}\right)-\Sigma_{x_{0}}\left(x_{1}\right) \geqq & 2 \int_{x_{1}}^{x_{2}} \omega^{2} \psi\left(\psi y^{\prime}-\psi^{\prime} y\right)\left(y^{\prime \prime}+y F\left(y^{2}, x\right)\right) d x \\
& +\frac{1}{2} \int_{x_{1}}^{x_{2}}\left(\omega^{2}\left(\psi^{2}\right)^{m}+\left(\omega^{2}\right)^{\prime} \psi \psi^{\prime \prime}\right) y^{2} d x .
\end{aligned}
$$

This relation obviously is still valid when $F$ is not $C^{1}$ as can be seen by approximating $F$ (with $\omega(x), \psi(x)$ and $y(x)$ fixed) by smooth functions. Upon taking $y$ to be a solution of (1) the first assertion of the lemma follows. The second assertion is proved similarly; note that condition (ii) implies $Q\left(y^{2}(x), x\right) \leqq 0$.

3. An oscillation criterion for the sublinear case. We assume here that $F$ in (1) satisfies (7). We assume moreover, as in the previous section, that $p(x)$ is positive and continuous on $(0, \infty)$, and that $(11)$ is nonoscillatory.

THEOREM 1. Assume that: there exists a $\lambda>0$ such that

(19) $z^{\prime \prime}+(1+\lambda) p(x) z=0$

is oscillatory; there exist positive functions $\omega \in C^{2}(0, \infty), \psi \in C^{3}(0, \infty)$, with $\psi$ a solution of (11), and such that for $x>0$

(20) $\omega^{\prime} \leqq 0$,

(21) $\omega^{2}\left(\psi^{2}\right)^{m}+\left(\omega^{2}\right)^{\prime} \psi \psi^{\prime \prime} \leqq 0$,

(22) $-\omega^{2} \psi^{3} \psi^{\prime \prime}=p \omega^{2} \psi^{4} \geqq c_{1}>0$;

there exist $M_{0}, x_{0}>0$ such that

(23) $F(t, x) \geqq\left(1+\lambda^{\prime}\right) p(x), x>x_{0}, t \leqq M_{0}^{2} \psi^{2}(x)$,

where $\lambda^{\prime} \geqq \max \left(\lambda, K^{-1}-1\right)$ ( $K$ is the constant in (7)); the function $\omega^{2} \psi^{2} G\left(\alpha^{2} \psi^{2}, x\right)$ is nonincreasing in $x$ for every $\alpha>0$.

Then (1) has oscillatory solutions.

Proof. Notice first that Lemma 4 and the hypothesis above imply that $\Sigma_{x_{0}}(x)$ is a nonincreasing function of $x$, for any solution $y$ of (1). Let $x_{0}>0$ and choose 
a solution $y$ of (1) with $y\left(x_{0}\right)=0,\left(y^{\prime}\left(x_{0}\right)\right)^{2}>c_{1} M_{0}^{2}\left(K\left(1+\lambda^{\prime}\right)-1\right) / \omega^{2}\left(x_{0}\right) \psi^{2}\left(x_{0}\right)$. Such a solution of (1) must satisfy

$$
y(x) \leqq M_{0} \psi(x) \text { for } x \geqq x_{0} .
$$

To see this, suppose there exists $x_{1} \geqq x_{0}$ with $y\left(x_{1}\right)=M_{0} \psi\left(x_{1}\right)$, then, because of (13) and (21), $\Sigma_{x_{0}}\left(x_{1}\right) \geqq \omega^{2} \psi \psi^{\prime \prime} y^{2}+\omega^{2} \psi^{2} G\left(y^{2}, x\right)$. Using (7) and the fact that $\psi$ satisfies (11),

$$
\begin{aligned}
\Sigma_{x_{0}}\left(x_{1}\right) & \geqq \omega^{2} \psi^{2} y^{2}\left(-p+K F\left(y^{2}, x\right)\right) \geqq \omega^{2} \psi^{2} y^{2}\left(-p+K F\left(M_{0}^{2} \psi^{2}\left(x_{1}\right), x\right)\right) \\
& \geqq \omega^{2} \psi^{2} y^{2} p\left(K\left(1+\lambda^{\prime}\right)-1\right) \geqq p M_{0}^{2} \omega^{2} \psi^{4}\left(K\left(1+\lambda^{\prime}\right)-1\right) \\
& \geqq c_{1} M_{0}^{2}\left(K\left(1+\lambda^{\prime}\right)-1\right) .
\end{aligned}
$$

However this implies

$$
\Sigma_{x_{0}}\left(x_{1}\right) \geqq \omega^{2}\left(x_{0}\right) \psi^{2}\left(x_{0}\right)\left(y^{\prime}\left(x_{0}\right)\right)^{2}=\Sigma_{x_{0}}\left(x_{0}\right),
$$

which is a contradiction, thus $y$ satisfies (24). Now (24), (23) and Lemma 3 together imply that $y$ is an oscillatory solution.

If one chooses $\omega(x) \equiv 1, \psi(x)=x^{1 / 2}$ and $p(x)=1 /\left(4 x^{2}\right)$, then (20), (21) and (22) are satisfied and (19) is oscillatory for any $\lambda>0$. More generally, we can take

(25) $\omega(x)=(\log x)^{\mu}$,

(26) $\psi(x)=x^{1 / 2}(\log x)^{-\delta}$,

(27) $p(x)=\left(1 / 4 x^{2}\right)\left(1-4 \delta(1+\delta)(\log x)^{-2}\right)$.

If $p$ is given by (27), then (19) will be oscillatory for every $\lambda>0$, and (20) will hold provided $\mu \leqq 0$. For the above choice of $\omega, \psi$ and $p$,

$$
p \omega^{2} \psi^{4}=\frac{1}{4}(\log x)^{2 \mu-4 \delta}\left(1-4 \delta(1+\delta)(\log x)^{-2}\right),
$$

so that (22) will hold, at least for large $x$, provided $\mu \geqq 2 \delta$.

Finally a computation yields

$$
\begin{aligned}
& \omega^{2}\left(\psi^{2}\right)^{m}+\left(\omega^{2}\right)^{\prime} \psi \psi^{\prime \prime} \\
& \quad=x^{-2}(\log x)^{2 \mu-2 \delta-1}\left(\frac{1}{2}(4 \delta-\mu)-2 \delta(1+\delta)(2(2 \delta+1)-\mu)(\log x)^{-2}\right),
\end{aligned}
$$

so that (21) will hold provided $4 \delta<\mu$. Thus if

(28) $2 \delta \leqq \mu \leqq 0$,

and $\omega$ and $\psi$ are given by (25) and (26) respectively, then (20), (21) and (22) will be satisfied. We thus have the following corollary to Theorem 1 .

Corollary 1. Let $\mu, \delta$ be real numbers satisfying (28). Assume that there exist $M_{0}, x_{0}>0$ such that

$$
F(t, x) \geqq(1+\lambda) / 4 x^{2}, \quad x>x_{0}, \quad t \leqq M_{0}^{2} x(\log x)^{-2 \delta},
$$

where $\lambda>K^{-1}-1$, and that the function $x(\log x)^{2(\mu-\delta)} G\left(\alpha^{2} x(\log x)^{-2 \delta}, x\right)$ is nonincreasing in $x$ for every $\alpha>0$. Then (1) has oscillatory solutions.

Under the hypothesis of Theorem 1, equation (1) may or may not also have nonoscillatory solutions. If (1) is linear, for example, then there are no nontrivial 
nonoscillatory solutions. The following result gives a condition not necessarily inconsistent with the hypothesis of Theorem 1 under which (1) will possess nontrivial nonoscillatory solutions.

THEOREM 2. Let $\psi$ be positive on $(0, \infty)$ and satisfy (11) and suppose that there exist $M_{1}, x_{1}>0$ such that

$$
F(t, x) \leqq p(x), \quad x>x_{1}, \quad t \geqq M_{1}^{2} \psi^{2}(x) .
$$

Then (1) has nontrivial nonoscillatory solutions.

Proof. Choose $y\left(x_{1}\right) \geqq M_{1} \psi\left(x_{1}\right),(y / \psi)^{\prime}\left(x_{1}\right)>0$. Observe that

$$
\left(\psi^{2}(y / \psi)^{\prime}\right)^{\prime}=-\left(\psi F+\psi^{\prime \prime}\right) y=\psi y(p-F) \geqq 0,
$$

and thus $(y / \psi)^{\prime}(x) \geqq(y / \psi)^{\prime}\left(x_{1}\right)>0, x \geqq x_{1}$. Such a solution then satisfies $y(x)$ $\geqq M_{1} \psi(x)$, for $x \geqq x_{1}$, and consequently, by (30) and Lemma 2, $y(x)$ is nonoscillatory.

REMARK 1. The hypothesis of Theorem 2 is sufficient to guarantee that all solutions of (1) have an unbounded right maximal interval of existence. (See Appendix.)

Applying Corollary 1 and Theorem 2 to equation (2) we obtain the following result.

COROLlaRY 2. Let $0<\gamma<1$, and let $q(x) x^{(\gamma+3) / 2}(\log x)^{\beta}$ be a nonincreasing function of $x$ with

$$
\lim _{x \rightarrow \infty} q(x) x^{(\gamma+3) / 2}(\log x)^{\beta}=k>0,
$$

for some $\beta \leqq 0$. Then (2) has oscillatory solutions as well as nontrivial nonoscillatory solutions.

Proof. Equation (2) is a special case of (1) with

(32) $F(t, x)=q(x) t^{(\gamma-1) / 2}$, and

(33) $G(t, x)=(2 /(1+\gamma)) q(x) t^{(\gamma+1) / 2}$.

The inequality (7) thus holds, in this case, with $K=1$. Now let $\mu=2 \delta=2 \beta /(1-\gamma)$. Then $\mu$ and $\delta$ satisfy (28) and, for $G(t, x)$ given by (33),

$$
x(\log x)^{2(\mu-\delta)} G\left(\alpha x(\log x)^{-2 \delta}, x\right)=\alpha^{(\gamma+1) / 2}(2 /(1+\gamma)) q(x) x^{(\gamma+3) / 2}(\log x)^{\beta} .
$$

Moreover, for $F(t, x)$ defined by (32) if $t \leqq M_{0}^{2} x(\log x)^{-2 \delta}$, then

$$
F(t, x) \geqq M_{0}^{\gamma-1} x^{(\gamma-1) / 2}(\log x)^{\beta} \geqq k M_{0}^{\gamma-1} x^{-2} .
$$

Thus if we take $M_{0}<(4 k)^{1 /(1-\gamma)}$, then (29) will hold for some $\lambda>0$. The existence of oscillatory solutions of (2) follows from Corollary 1.

To prove the existence of nonoscillatory solutions of (2), choose, by (31), an $x_{1}>0$ such that

$$
q(x) x^{(\gamma+3) / 2}(\log x)^{\beta}<2 k \text { for } x \geqq x_{1} .
$$


If $x \geqq x_{1}$ and $t \geqq M_{1}^{2} x(\log x)^{-2 \delta}$ then

$$
\begin{aligned}
F(t, x) & =q(x) t^{(\gamma-1) / 2} \leqq M_{1}^{\gamma-1} x^{(\gamma-1) / 2}(\log x)^{\beta} \\
& \leqq 2 k M_{1}^{-1} x^{-2}
\end{aligned}
$$

Thus if we choose $M_{1}>(8 k)^{1 /(1-\gamma)}$, then $F$ will satisfy (30), with $\psi$ given by (26), and the existence of nonoscillatory solutions of (2) follows from Theorem 2.

For $\beta=0$ this result was proved by Belohorec [2].

4. A nonoscillation criterion in the sublinear case. Like the theorems in $\S 3$, this result is also motivated by a theorem of Belohorec [2]. The extension in this case seems somewhat deeper than that of Theorems 1 and 2.

THEOREM 3. Let $F(t, x)$ satisfy (5); assume that

$$
y_{1} F\left(y_{1}^{2}, x\right) \leqq y_{2} F\left(y_{2}^{2}, x\right) \text { for } y_{2} \geqq y_{1} \text {. }
$$

In addition to the hypothesis of Theorem 2, assume that $\psi \in C^{3}(0, \infty)$ satisfies

$$
\omega(x)=\int^{x} \frac{d x}{\psi^{2}(x)} \rightarrow \infty \text { as } x \rightarrow \infty,
$$

and

$$
\omega^{2}\left(\psi^{2}\right)^{m}+\left(\omega^{2}\right)^{\prime} \psi \psi^{\prime \prime} \geqq 0 .
$$

Moreover, assume that for every $\alpha>0, \omega^{2} \psi^{2} G\left(\alpha^{2} \psi^{2}, x\right)$ is a nondecreasing function of $x$, and that

$$
\lim _{x \rightarrow \infty} \omega^{2} \psi^{2} G\left(\alpha^{2} \psi^{2}, x\right)=k(\alpha)<\infty
$$

Then equation (1) is nonoscillatory on $\left[x_{1}, \infty\right)$.

Proof. We observe first that if $y$ is a solution of (1) then $\Sigma_{x_{0}}(x)$, defined by (13), is a nondecreasing function of $x$. This follows from the assumption concerning $G$, (36) and inequality (14) of Lemma 4.

We point out that, in view of the above observation, any possibility of pathological behavior of solutions of (1) is completely ruled out under the hypothesis of Theorem 3. Regarding the question of global existence, see Remark 1 following the proof of Theorem 2. In fact, because of (30), the zeros of a solution $y$ of (1) can have a cluster point at $x=a<\infty$ only if

$$
\limsup _{x \rightarrow a}\left|y^{\prime}(x)\right|=0
$$

but in view of the nondecreasing character of $\Sigma$, and because $a$ is a right cluster point of zeros of $y$, it is clear from (13) that this is impossible.

We shall now assume that there exists an oscillatory solution $y(x)$ of (1) and show that this assumption leads to a contradiction. If $x_{1}, M_{1}$ are as in Theorem 2 , and $y$ is an oscillatory solution of (1), then there must exist an $x_{2} \geqq x_{1}$ such that

$$
|y(x)| \leqq M_{1} \psi(x), \quad x \geqq x_{2} .
$$


For otherwise, since $y$ is oscillatory, there would exist $x_{3} \geqq x_{1}$ with

$$
|y(x)|=M_{1} \psi(x), \quad\left|(y / \psi)^{\prime}\right|>0, \quad \text { at } x=x_{3} .
$$

As the proof of Theorem 2 shows, a solution of (1) satisfying (39) could not change sign for $x \geqq x_{3}$, and this contradicts the assumption that $y$ is oscillatory.

We put

$$
\eta=\omega\left(\psi y^{\prime}-\psi^{\prime} y\right)=\omega \psi^{2}(y / \psi)^{\prime},
$$

and observe that since $\omega^{2} \psi \psi^{\prime \prime} y^{2}=-p \omega^{2} \psi^{2} y^{2} \leqq 0$, then in view of (13) and the nondecreasing character of $\Sigma, \eta^{2}(x) \geqq \Sigma_{x_{0}}(x)-\omega^{2} \psi^{2} G\left(y^{2}, x\right)$, for any $x_{0}>0$. Since $F$ is positive, $G$ is nondecreasing in its first argument, so by (38),

$$
\eta^{2}(x) \geqq \Sigma_{x_{0}}(x)-\omega^{2} \psi^{2} G\left(M_{1}^{2} \psi^{2}, x\right), \quad x \geqq x_{2},
$$

and finally, using (37) and the nondecreasing character of $\omega^{2} \psi^{2} G\left(M_{1}^{2} \psi^{2}, x\right)$,

$$
\eta^{2}(x) \geqq \Sigma_{x_{0}}(x)-k, \quad x \geqq x_{2},
$$

where here and below, $k=k\left(M_{1}^{2}\right)$. For the oscillatory solution $y$, it follows from Rolle's theorem and (40) that there exist arbitrarily large values of $x$ for which $\eta(x)=0$. Thus from (41) and the nondecreasing character of $\Sigma$ there follows, for $x_{0}>0$,

$$
\Sigma_{x_{0}}\left(x_{0}\right) \leqq k, \quad x \geqq x_{2} .
$$

Since $\Sigma_{x_{0}}\left(x_{0}\right)=\eta^{2}\left(x_{0}\right), \Sigma_{x_{0}}(x) \geqq 0$ for $x \geqq x_{0}$, thus, as $\omega^{2} \psi \psi^{\prime \prime} y^{2} \leqq 0$, we have, from (13),

$$
\int_{x_{0}}^{x}\left(\psi y^{\prime}-\psi^{\prime} y\right)^{2}\left(\omega^{2}\right)^{\prime} d x \leqq \eta^{2}(x)+\omega^{2} \psi^{2} G\left(y^{2}, x\right),
$$

for $x \geqq x_{0}$. Using (37) and (38) as before we obtain

$$
\int_{x_{0}}^{x}\left(\psi y^{\prime}-\psi^{\prime} y\right)^{2}\left(\omega^{2}\right)^{\prime} d x \leqq \eta^{2}(x)+k, \quad x \geqq x_{2},
$$

and finally, since $\omega^{\prime}>0$, and since $\eta$ vanishes for certain arbitrarily large values of $x$,

$$
\int_{x_{0}}^{\infty}\left(\psi y^{\prime}-\psi^{\prime} y\right)^{2}\left(\omega^{2}\right)^{\prime} d x \leqq k .
$$

We wish to show next that $\eta$ is bounded. From (13) and (40), using (42) and (43), we obtain

$$
\eta^{2}(x) \leqq 2 k-\omega^{2} \psi \psi^{\prime \prime} y^{2}-\omega^{2} \psi^{2} G\left(y^{2}, x\right), \quad x \geqq x_{2} .
$$

Since (5) implies (7) with $K=1$, we have

$$
\begin{aligned}
\eta^{2}(x) & \left.\leqq 2 k-\omega^{2} \psi \psi^{\prime \prime} y^{2}-\omega^{2} \psi^{2} y^{2} F^{\prime} y^{2}, x\right), & & x \geqq x_{2}, \\
& \leqq 2 k-\omega^{2} \psi^{2} y^{2}\left(F\left(y^{2}, x\right)-p(x)\right), & & x \geqq x_{2} .
\end{aligned}
$$


At a zero of $\eta^{\prime}$ we have

$$
0=\eta^{\prime}(x)=-\omega y \psi\left(F\left(y^{2}, x\right)-p(x)\right)+\left(\omega^{\prime} / \omega\right) \eta,
$$

so at such a value $x \geqq x_{2}$,

$$
\eta^{2} \leqq 2 k-\psi y \omega^{\prime} \eta
$$

Now using (35) and (38), we find that, for $x \geqq x_{2}, \eta^{\prime}(x)=0$ implies $\eta^{2}(x) \leqq 2 k$ $+M_{1}|\eta(x)|$, and this implies that the extremal values of $\eta$ are uniformly bounded, hence $\eta(x)$ is bounded as $x \rightarrow \infty$, say

$$
|\eta(x)| \leqq B
$$

Now for $y(x)>0$ we have

$$
\begin{aligned}
\eta^{\prime}(x) & =\omega \psi y\left(p(x)-F\left(y^{2}, x\right)\right)+\left(\omega^{\prime} / \omega\right) \eta, \\
& \geqq-\omega \psi y F\left(y^{2}, x\right)+\left(\omega^{\prime} / \omega\right) \eta .
\end{aligned}
$$

If $x \geqq x_{2}$, then using (35), (38) and (34), we obtain

$$
\begin{aligned}
\omega \psi y F\left(y^{2}, x\right) & =\left(\omega^{\prime} / \omega\right) \omega^{2} \psi^{3} y F\left(y^{2}, x\right), \\
& \leqq M_{1}\left(\omega^{\prime} / \omega\right) \omega^{2} \psi^{4} F\left(M_{1}^{2} \psi^{2}, x\right),
\end{aligned}
$$

from which there follows, with the use of (7),

$$
\begin{aligned}
\omega \psi y F\left(y^{2}, x\right) & \leqq M_{1}^{-1}\left(\omega^{\prime} / \omega\right) \omega^{2} \psi^{2} G\left(M_{1}^{2} \psi^{2}, x\right), \\
& \leqq K M_{1}^{-1} k\left(\omega^{\prime} / \omega\right) .
\end{aligned}
$$

Thus for $y(x)>0, x \geqq x_{2}$, by use of the above and (45), we find

$$
\begin{aligned}
\eta^{\prime}(x) & \geqq-\left(k K M_{1}^{-1}+B\right)\left(\omega^{\prime} / \omega\right), \\
& \geqq-B_{1}\left(\omega^{\prime} / \omega\right) .
\end{aligned}
$$

Now take $x_{n}$ to be a zero of $y$ with $y^{\prime}\left(x_{n}\right)>0$, and let $\bar{x}_{n}$ be the first zero of $\eta$ to the right of $x_{n}$, so that $y(x), \eta(x) \geqq 0$ on $\left[x_{n}, \bar{x}_{n}\right]$. Since (46) holds on $\left[x_{n}, \bar{x}_{n}\right]$,

$$
0=\eta\left(\bar{x}_{n}\right) \geqq \eta\left(x_{n}\right)-B_{1} \int_{x_{n}}^{x_{n}}\left(\omega^{\prime} / \omega\right) d x,
$$

and thus we can choose $\xi_{n}, x_{n}<\xi_{n}<\bar{x}_{n}$, such that

$$
\int_{x_{n}}^{\xi_{n}}\left(\omega^{\prime} / \omega\right) d x=\frac{1}{2} B_{1}^{-1} \eta\left(x_{n}\right)
$$

But then for $x_{n} \leqq x \leqq \xi_{n}$,

$$
\eta(x) \geqq \eta\left(x_{n}\right)-B_{1} \int_{x_{n}}^{\xi_{n}}\left(\omega^{\prime} / \omega\right) d x=\frac{1}{2} \eta\left(x_{n}\right) .
$$

Consequently,

$$
\int_{x_{n}}^{\xi_{n}} \eta^{2}\left(\omega^{\prime} / \omega\right) d x \geqq B_{1}^{-1}\left(\frac{1}{2} \eta\left(x_{n}\right)\right)^{3}
$$


If $x_{0}$ is the first zero of $y$ then using (13), (40) and the nondecreasing character of $\Sigma_{x_{0}}$, we obtain

$$
\begin{aligned}
\eta^{2}\left(x_{n}\right) & \geqq \eta^{2}\left(x_{0}\right)+\int_{x_{0}}^{x_{n}} \eta^{2}\left(\omega^{\prime} / \omega\right) d x, \\
& \geqq \eta^{2}\left(x_{0}\right) .
\end{aligned}
$$

Thus

$$
\int_{x_{n}}^{\xi_{n}} \eta^{2}\left(\omega^{\prime} / \omega\right) d x \geqq B_{1}^{-1}\left(\frac{1}{2} \eta\left(x_{0}\right)\right)^{3} .
$$

Since $x_{n}$ was an arbitrary zero of $y,(44)$ and (47) contradict the assumption that $y$ is an oscillatory solution of (1). This completes the proof of Theorem 3.

Remark 2. Condition (5), or what is its consequence, (7), with $K=1$ is used only in the proof that $\eta$ is bounded. If we assume that $\left|\omega^{2} \psi^{3} \psi^{\prime \prime}\right|$ is bounded for large $x$, then in the hypothesis of Theorem 3, (5) can be weakened to (7) with $0<K$.

One choice for $\psi$ in Theorem 3 is

$$
\psi(x)=x^{(1-\varepsilon) / 2}, \quad 0<\varepsilon<1 .
$$

It is clear that $\omega$ can be allowed to differ from an indefinite integral of $\psi^{-2}$ by a positive constant factor, accordingly we take

$$
\omega(x)=x^{\varepsilon} .
$$

The inequality (36) is easily verified for this choice of $\psi$ and $\omega$; moreover, $\omega^{2} \psi^{3} \psi^{\prime \prime}$ $=-\frac{1}{4}\left(1-\varepsilon^{2}\right)$, so that in view of the remark following the proof of Theorem 3 , for this choice of $\psi,(5)$ in the hypothesis of that theorem can be replaced by (7) with $0<K$. The coefficient $p$ in (11) corresponding to $\psi(x)=x^{(1-\varepsilon) / 2}$ is $\left(1-\varepsilon^{2}\right) / 4 x^{2}$.

A second choice for $\omega, \psi$ and $p$ is (25), (26), (27) with

$$
\delta \geqq \frac{1}{2}, \quad \mu=2 \delta+1 .
$$

From $\left(27^{\prime}\right)$ and (50) one can readily verify (36), and, except for a positive constant factor, $\omega$ is an indefinite integral of $\psi^{-2}$. We have thus the following two corollaries to Theorem 3.

CoRollary 3. Let $F(t, x)$ satisfy (7) and (34). Let $1>\varepsilon>0$, and assume that, for every $\alpha>0, x^{1+\varepsilon} G\left(\alpha x^{1-\varepsilon}, x\right)$ is nondecreasing with a finite upper bound, and that there exist $x_{1}, M_{1}>0$ such that $F(t, x) \leqq\left(1-\varepsilon^{2}\right) / 4 x^{2}$ for $t \geqq M_{1}^{2} x^{1-\varepsilon}$. Then equation (1) is nonoscillatory on $\left(x_{1}, \infty\right)$.

Corollary 4. Let $F(t, x)$ satisfy (5) and (34). Let $\delta>\frac{1}{2}$ and suppose that, for every $\alpha>0, x(\log x)^{2 \delta+2} G\left(\alpha x(\log x)^{-2 \delta}, x\right)$ is nondecreasing with a finite upper bound. Moreover, assume that there exist $x_{1}, M_{1}>0$ such that

$$
F(t, x) \leqq\left(1-4 \delta(1+\delta)(\log x)^{-2}\right) / 4 x^{2}, \quad t \geqq M_{1}^{2} x(\log x)^{-2 \delta} .
$$

Then equation (1) is nonoscillatory on $\left(x_{1}, \infty\right)$. 
Specializing Corollaries 3 and 4 to equation (2) we obtain the following results.

Corollary 5 (Belohorec [2]). Let $0<\gamma<1, q(x) \geqq 0$, and assume that, for some $\beta, 0<\beta<(1-\gamma) / 2, x^{(3+\gamma) / 2+\beta} q(x)$ is nondecreasing and bounded above. Then (2) is nonoscillatory.

COROllaRY 6. Let $0<\gamma<1, q(x) \geqq 0$, and assume that, for some $\beta, \beta \geqq(5-\gamma) / 2$, $q(x) x^{(3+y) / 2}(\log x)^{\beta}$ is nondecreasing and bounded above. Then (2) is nonoscillatory.

5. A oscillation criterion for the superlinear equation. We assume in this section that $F$ satisfies (6) for some $K \geqq 1$, and we assume as before that (11), with $p$ positive and continuous on $(0, \infty)$, is nonoscillatory, and that there exists an $\varepsilon>0$ such that

(51) $y^{\prime \prime}+(1+\varepsilon) p(x) y=0$

is oscillatory. Moreover, we assume that $\psi \in C^{3}(0, \infty), \psi>0$, is a solution of (11) and that

(52) $\left(\psi^{2}\right)^{m} \geqq 0$,

(53) $\int^{\infty} d x / \psi^{2}(x)=\infty$,

(54) $-\psi^{\prime \prime} \psi^{3}=p \psi^{4} \leqq C_{0}<\infty$.

Finally we assume that for every $\alpha>0, \psi^{2} G\left(\alpha^{2} \psi^{2}(x), x\right)$ is a nondecreasing function of $x$, and that there exist $M_{0}, x_{0}>0$ and $\varepsilon^{\prime}>\varepsilon$ such that

(55) $F(t, x) \geqq\left(1+\varepsilon^{\prime}\right) p(x), x \geqq x_{0}, t \geqq M_{0}^{2} \psi^{2}(x)$.

The proof of the superlinear oscillation theorem will be based on the following sequence of lemmas. It will be assumed throughout this section that the conditions stated in the preceding paragraph are fulfilled. We first reformulate Lemma 2 for $r(x)=(1+\varepsilon) p(x)$.

LEMMA $2^{\prime}$. If $y(x)$ is a nonoscillatory solution of $(1)$, then

$$
\liminf _{x \rightarrow \infty}(p(x))^{-1} F\left(y^{2}(x), x\right) \leqq 1+\varepsilon .
$$

LEMMA 5. If $y(x)$ is a nonoscillatory solution of $(1)$ and $(y / \psi)^{\prime}$ is eventually of one sign, then

$$
\lim _{x \rightarrow \infty}\left(y^{\prime} \psi-\psi^{\prime} y\right)(x)=0 .
$$

Proof. First we shall show that

$$
\left|\int^{\infty}(y / \psi)^{\prime} d x\right|<\infty
$$

If $y$ is eventually positive and $(y / \psi)^{\prime}$ is eventually negative, this is clear. If $y$ and $(y / \psi)^{\prime}$ are both eventually positive, then because of (55) and (56), we must have $y \leqq M_{0} \psi(x)$ for all large $x$ and thus (58) follows in this case also.

Since we can assume then in either case that $0<y(x) \leqq M_{0} \psi(x)$, for all large $x$, we have $\left(y^{\prime} \psi-y \psi^{\prime}\right)^{\prime}=y^{\prime \prime} \psi-y \psi^{\prime \prime} \leqq-M_{0} \psi \psi^{\prime \prime}$, so from (54)

$$
\left(y^{\prime} \psi-y \psi^{\prime}\right)^{\prime} \leqq C_{0} M_{0} \psi^{-2} \text {, }
$$


for all large $x$. Consider now the case where $(y / \psi)^{\prime}$ is eventually positive. We shall suppose that

$$
0<Q \leqq \limsup _{x \rightarrow \infty}\left(y^{\prime} \psi-y \psi^{\prime}\right)(x),
$$

and show that this leads to a contradiction. We first choose $A$ so that

$$
\int_{A}^{\infty}\left|(y / \psi)^{\prime}\right| d x<\frac{Q^{2}}{32 C_{0} M_{0}}
$$

Because of (60) and (53) we can choose $\xi_{0}, \xi_{1}$ so that $A \leqq \xi_{0} \leqq \xi_{1},\left(y^{\prime} \psi-y \psi^{\prime}\right)\left(\xi_{1}\right) \geqq \frac{1}{2} Q$, and $C_{0} M_{0} \int_{\xi_{0}}^{\xi_{1}} \psi^{-2}(x) d x=\frac{1}{4} Q$. Then from (59)

$$
\begin{aligned}
\left(y^{\prime} \psi-y \psi^{\prime}\right)(x) & \geqq\left(y^{\prime} \psi-y \psi^{\prime}\right)\left(\xi_{1}\right)-C_{0} M_{0} \int_{\xi_{0}}^{\xi_{1}} \psi^{-2}(x) d x, \\
& \geqq \frac{1}{4} Q,
\end{aligned}
$$

for $\xi_{0} \leqq x \leqq \xi_{1}$. But this gives

$$
\int_{\xi_{0}}^{\xi_{1}}(y / \psi)^{\prime} d x \geqq \frac{1}{4} Q \int_{\xi_{0}}^{\xi_{1}} \psi^{-2} d x=\frac{Q^{2}}{16 C_{0} M_{0}}
$$

which, since $\xi_{0}>A$, contradicts (61). The case where $y$ and $(y / \psi)^{\prime}$ eventually have opposite sign is handled similarly.

We now take $\omega \equiv 1$ in (13), then from (52) and the nondecreasing character of $\psi^{2} G\left(\alpha^{2} \psi^{2}, x\right)$, it follows that $\Sigma$ is a nondecreasing function of $x$.

LEMMA 6. Let $y$ be a nonoscillatory solution of (1), then there exists a sequence $\xi_{n}$ such that $\lim _{n \rightarrow \infty} \xi_{n}=\infty$,

$$
F\left(y^{2}\left(\xi_{n}\right), \xi_{n}\right) \leqq(1+\varepsilon) p\left(\xi_{n}\right), \quad n=1,2, \ldots,
$$

and

$$
\lim _{n \rightarrow \infty} \Sigma\left(\xi_{n}\right) \leqq C_{0}(K(1+\varepsilon)-1) \limsup _{n \rightarrow \infty}\left[(y / \psi)\left(\xi_{n}\right)\right]^{2}
$$

Proof. Suppose that the hypothesis of Lemma 5 is satisfied, so that (57) holds. Because of Lemma $2^{\prime}$ it is possible to choose $\left\{\xi_{n}\right\}$ so that (62) is satisfied. It follows then from (13) and (57) that

$$
\lim _{n \rightarrow \infty} \Sigma\left(\xi_{n}\right) \leqq \limsup _{n \rightarrow \infty}\left\{\left(\psi \psi^{\prime \prime} y^{2}\right)\left(\xi_{n}\right)+\psi^{2}\left(\xi_{n}\right) G\left(y^{2}\left(\xi_{n}\right), \xi_{n}\right)\right\} .
$$

Using (6) followed by (62) and (54), we obtain

$$
\begin{aligned}
\psi\left(\xi_{n}\right) \psi^{\prime \prime}\left(\xi_{n}\right) y^{2}\left(\xi_{n}\right) & +\psi^{2}\left(\xi_{n}\right) G\left(y^{2}\left(\xi_{n}\right), \xi_{n}\right) \\
& \leqq\left(y\left(\xi_{n}\right) / \psi\left(\xi_{n}\right)\right)^{2}\left[\psi^{3}\left(\xi_{n}\right) \psi^{\prime \prime}\left(\xi_{n}\right)+K \psi^{4}\left(\xi_{n}\right) F\left(y^{2}\left(\xi_{n}\right), \xi_{n}\right)\right] \\
& \leqq C_{0}[K(1+\varepsilon)-1]\left(y\left(\xi_{n}\right) / \psi\left(\xi_{n}\right)\right)^{2}
\end{aligned}
$$

In view of (64), this last inequality implies (63). 
If the hypothesis of Lemma 5 does not hold, i.e. if $(y / \psi)^{\prime}$ changes sign infinitely often, then we choose $\left\{\xi_{n}\right\}$ so that $\xi_{n} \rightarrow \infty$, and, for $n=1,2, \ldots$,

(65) $\left(y^{\prime} \psi-\psi^{\prime} y\right)\left(\xi_{n}\right)=0$, and

(66) $\left(y^{\prime} \psi-\psi^{\prime} y\right)^{\prime}\left(\xi_{n}\right) \geqq 0$.

But $\left(y^{\prime} \psi-\psi^{\prime} y\right)^{\prime}=-\psi y\left(F\left(y^{2}, x\right)-p\right)$, so that, for $y>0$, (66) implies (62) with $\varepsilon=0$. We assume, as we can without loss of generality, that $y(x)>0$ for all large $x$; then (65) implies (64), and we obtain (63) from (64) as before.

THEOREM 4. Under the conditions imposed in the first paragraph of this section, equation (1) has oscillatory solutions.

Proof. In view of (55) and (62),

$$
\limsup _{n \rightarrow \infty}\left[(y / \psi)\left(\xi_{n}\right)\right]^{2} \leqq M_{0}^{2} .
$$

Thus, since $\Sigma$ is nondecreasing, it follows from Lemma 6 that for any nonoscillatory solution $y$ of (1), $\Sigma$, defined by (13), satisfies

$$
\lim _{x \rightarrow \infty} \Sigma(x) \leqq C_{0} M_{0}^{2}(K(1+\varepsilon)-1) .
$$

Again because of the monotonicity of $\Sigma$, if $y$ is a solution of (1) with $y\left(x_{0}\right)=0$ and $y^{\prime}\left(x_{0}\right)$ satisfying $\left(\eta\left(x_{0}\right)\right)^{2}=\left(\psi\left(x_{0}\right) y^{\prime}\left(x_{0}\right)\right)^{2}>C_{0} M_{0}^{2}(K(1+\varepsilon)-1)$, where $x_{0}$ is sufficiently large, then (67) cannot hold, and $y$ must therefore be oscillatory.

As a corollary of the proof of Theorem 4 we have the following result.

COROllary 7. If (6) holds with $K=1$, in particular if (4) holds, and if (51) is oscillatory for every $\varepsilon>0$, then under the conditions of Theorem 4 every solution of (1) with at least one zero in $(0, \infty)$ is oscillatory.

Proof. Under the above conditions $\varepsilon$ can be taken arbitrarily in (62) and hence in (67). Thus if (6) holds with $K=1$, (67) shows that, since $\Sigma$ is nondecreasing, $\Sigma(x) \leqq 0$ for a nonoscillatory solution of (1). On the other hand, if $y$ is a nontrivial solution of (1) and $y\left(x_{0}\right)=0$, then $\Sigma(x) \geqq \Sigma\left(x_{0}\right)>0$ for $x>x_{0}$; thus $y$ must be oscillatory.

COROLlaRy 8. If $\psi^{2}(x) G\left(\alpha^{2} \psi^{2}(x), x\right) \rightarrow \infty$, as $x \rightarrow \infty$ for every $\alpha>0$, then under the conditions of Theorem 4 every solution of $(1)$ with at least one zero in $(0, \infty)$ is oscillatory.

Proof. Because of (55), it is implied by (62) that

(68) $y\left(\xi_{n}\right) \leqq M_{0} \psi\left(\xi_{n}\right)$,

but by (6), (54) and (62), when (68) holds,

$$
\begin{aligned}
\psi^{2}\left(\xi_{n}\right) G\left(y^{2}\left(\xi_{n}\right), \xi_{n}\right) & \leqq K \psi^{4}\left(\xi_{n}\right) F\left(y^{2}\left(\xi_{n}\right), \xi_{n}\right), \\
& \leqq K(1+\varepsilon) p\left(\xi_{n}\right) \psi^{4}\left(\xi_{n}\right), \\
& \leqq(1+\varepsilon) K C_{0} .
\end{aligned}
$$


We now claim that $\left(y\left(\xi_{n}\right) / \psi\left(\xi_{n}\right)\right)^{2} \rightarrow 0$. Suppose not, then there exists a subsequence $\left\{\xi_{n_{i}}\right\}$ such that $y^{2}\left(\xi_{n_{i}}\right) / \psi^{2}\left(\xi_{n_{i}}\right) \geqq \alpha^{2}>0$, for some $\alpha$. Observe that

$$
\psi^{2}\left(\xi_{n_{i}}\right) G\left(\alpha^{2} \psi^{2}\left(\xi_{n_{i}}\right), \xi_{n_{i}}\right) \leqq \psi^{2}\left(\xi_{n_{i}}\right) G\left(y^{2}\left(\xi_{n_{i}}\right), \xi_{n_{i}}\right)
$$

which is bounded, contradicting the given hypothesis that $\psi^{2}(x) G\left(\alpha^{2} \psi^{2}(x), x\right) \rightarrow \infty$ as $x \rightarrow \infty$ for every $\alpha>0$. Thus for a nonoscillatory solution of (1), (63) implies $\Sigma(x) \leqq 0$ and the result then follows as before.

If we take $\psi(x)=x^{1 / 2}$, then $\psi$ satisfies (11) with $p(x)=1 /\left(4 x^{2}\right)$. Moreover, one readily sees that (52), (53) and (54) are satisfied. Thus Theorem 4 and Corollaries 7 and 8 imply the following result.

Corollary 9. Let $F$ satisfy (6). Suppose that there exist constants $x_{0}, M, c>0$ such that $x^{2} F(t, x) \geqq \frac{1}{4}+c, x \geqq x_{0}, t \geqq M^{2} x$, and suppose that, for every $\alpha>0$, $x G(\alpha x, x)$ is nondecreasing in $x$. Then (1) has oscillatory solutions. If (6) holds with $K=1$, or if for every $\alpha>0, \lim _{x \rightarrow \infty} x G(\alpha x, x)=\infty$, then every solution of (1) with one zero in $(0, \infty)$ is oscillatory.

The first assertion, that is, the existence of oscillatory solutions of (1) under the hypothesis of Corollary 9, was given in [3, Theorem 1]. An examination of the derivation of inequality (63) of Lemma 6 shows that in Theorem 4 the hypothesis (6) can be weakened to $G(t, x) \leqq K F(t, x), t \leqq M^{2} \psi(x)$. With a similar modification of the hypothesis, Corollary 9 in fact contains Theorem 1 of [3].

If we specialize Corollary 9 to equation (2) we obtain the following result, which is a refinement of a theorem of Jasný and Kurzweil [7] and [9]:

COROLlaRY 10. Let $\gamma>1, q(x) \geqq 0$, and assume that $x^{(\gamma+3) / 2} p(x)$ is a nondecreasing function of $x$, then every nontrivial solution of (2) which vanishes at least once in $(0, \infty)$ is oscillatory.

6. Existence of nonoscillatory solutions in the superlinear case. Whereas in $\S 7$ to follow we shall give criteria for (1) to be nonoscillatory, i.e. to have no oscillatory solutions, in this section we shall discuss conditions which imply the existence of nontrivial nonoscillatory solutions of (1). More specifically, the main result of this section will give conditions consistent with those of Theorem 4 which imply the existence of nonoscillatory solutions of (1) with certain prescribed growth properties.

A necessary and sufficient condition for (2), with $\gamma>1$, to have nonoscillatory solutions has been given by Atkinson [1], and related results for the equation (1) have been given by Nehari [11] and Wong [15], as well as others; see the references in [15]. Some further discussion of these results seems to be in order here prior to the statement of our results in this direction. If $F(t, x)$ is monotone in $t$ for each fixed $x$ then the condition

$$
\int^{\infty} x F(c, x) d x<\infty, \text { for some } c>0,
$$


is necessary and sufficient for the existence of bounded nonoscillatory solutions, see Nehari [11, Theorem I], and for a similar result with a requirement on $F$ somewhat weaker than monotonicity in $t$, see Wong [15, Theorem 3]. For (2) with $\gamma>1$, Atkinson's theorem states that (69), which reduces to $\int^{\infty} x q(x) d x<\infty$, is necessary for the existence of any nonoscillatory solutions. Thus the class of equations of the form (2) with $\gamma>1$ has the property (P): the existence of any nonoscillatory solutions implies the existence of bounded ones. We make note here of some other classes of equations which also have the property (P). Nehari, [11], [12], has shown that equations of the form (1) with $F$ subject to the nonlinearity condition $(\mathrm{N})$ : there exists an $\varepsilon>0$ such that, for each $x>0,0<t_{1}^{-\varepsilon} F\left(t_{1}, x\right) \leqq t_{2}^{-\varepsilon} F\left(t_{2}, x\right)$ whenever $0<t_{1}<t_{2}<\infty$, share many of the distinctive properties of (2), $\gamma>1$. We shall show that this class of equations has also the property $(\mathrm{P})$. Indeed in the presence of the condition $(\mathrm{N}),(69)$ is necessary for the existence of nonoscillatory solutions of (1). This is in fact contained in [15, Theorem 4], nevertheless we give a proof of this special case here.

Proposition (*). Let $F$ satisfy condition $(\mathrm{N})$, then a necessary condition for (1) to possess nontrivial nonoscillatory solutions is (69).

Proof. Let $y$ be a nonoscillatory solution of (1) and suppose that $y(x)>0$ for $x>x_{0}$. Since $y$ is nonoscillatory, $\lim _{x \rightarrow \infty} y^{\prime}(x)=0$, and thus

$$
y^{\prime}(x)=\int_{x}^{\infty} y(t) F\left(y^{2}(t), t\right) d t
$$

so that, as $y$ is nondecreasing as well as positive for $x \geqq x_{0}$,

$$
\begin{aligned}
(y(x))^{-1-2 \varepsilon} y^{\prime}(x) & \geqq \int_{x}^{\infty}(y(t))^{-2 \varepsilon} F\left(y^{2}(t), t\right) d t \\
& \geqq \int_{x}^{\infty}\left(y\left(x_{0}\right)\right)^{-2 \varepsilon} F\left(y^{2}\left(x_{0}\right), t\right) d t .
\end{aligned}
$$

Denote $y^{2}\left(x_{0}\right)=c$ and integrate the above inequality to give

$$
\begin{aligned}
(2 \varepsilon)^{-1}\left\{\left(y\left(x_{0}\right)\right)^{-2 \varepsilon}-\left(y\left(x_{1}\right)\right)^{-2 \varepsilon}\right\} & \geqq \int_{x_{0}}^{x_{1}} \int_{x}^{\infty} c^{-\varepsilon} F(c, t) d t d x \\
& \geqq \int_{x_{0}}^{x_{1}} \int_{x}^{x_{1}} c^{-\varepsilon} F(c, t) d t d x \geqq \int_{x_{0}}^{x_{1}} \int_{x_{0}}^{t} c^{-\varepsilon} F(c, t) d x d t \\
& =c^{-\varepsilon} \int_{x_{0}}^{x_{1}}\left(t-x_{0}\right) F(c, t) d t,
\end{aligned}
$$

which shows, upon letting $x_{1} \rightarrow \infty$, that $F$ must satisfy (69). Moreover, if $y(x)$ is unbounded, then the integral in (69) must be finite for every $c>0$.

Since in the presence of $(\mathrm{N})$, condition (69) is sufficient for the existence of bounded nonoscillatory solutions of (1), Proposition (*) implies that the class of equations of the form (1) with $F$ satisfying the condition $(\mathrm{N})$ has the property $(\mathrm{P})$. 
If $\varepsilon$ in $(\mathrm{N})$ is allowed to equal zero, this is not true, as is shown by the example of the Euler equation $y^{\prime \prime}+\left(1 / 4 x^{2}\right) y^{\prime}=0$.

A larger class with the property $(\mathrm{P})$ is obtained by replacing the condition $(\mathrm{N})$ by the following condition $\left(\mathrm{N}^{\prime}\right): F(t, x)$ is monotone in $t$ for each fixed $x>0$ and there exist $p>1, T>0$ such that, for all $x>0,\left(\log t_{1}\right)^{-p} F\left(t_{1}, x\right) \leqq\left(\log t_{2}\right)^{-p} F\left(t_{2}, x\right)$ whenever $T<t_{1} \leqq t_{2}$. An analogue of $(*)$ for this case can be proved in exactly the same way as $(*)$. If $p$ is allowed to equal 1 in $\left(\mathrm{N}^{\prime}\right)$, then the resulting class again fails to have the property $(\mathrm{P})$. This is shown by the following example:

$$
y^{\prime \prime}+\frac{y \log \left(y^{2}+1\right)}{4 x^{2} \log (x+1)}=0,
$$

which has the nonoscillatory solution $x^{1 / 2}$, while the coefficient

$$
F(t, x)=\frac{\log (t+1)}{4 x^{2} \log (x+1)}
$$

fails to satisfy (69). We remark that Theorem 4 of [15] enables one to determine an even larger class of equations which has the property $(\mathrm{P})$.

We now wish to impose conditions which imply the existence of nonoscillatory solutions of (1) which are bracketed between two preassigned functions. Accordingly we assume the existence of functions $\psi(x), \varphi_{1}(x)$, and $\varphi_{3}(x)$ which are positive and continuous on $(0, \infty)$ and such that

(70) $G(t, x) \geqq p(x) t, t \geqq \varphi_{1}^{2}(x)$, and

(71) $p(x) \varphi_{3}^{2}(x)<-a \psi^{-2}(x), 0<x<\infty$, where $a<0$,

and look for nonoscillatory solutions of (1) for which the energy function $\Sigma(x)$, as defined by (13), satisfies

(72) $\Sigma(x) \leqq a<0$,

with $\omega(x) \equiv 1$. In fact, we establish in the following result the existence of nonoscillatory solutions which are bracketed between the functions $\varphi_{1}$ and $\varphi_{3}$.

THEOREM 5. Let $\psi \in C^{3}(0, \infty), \psi>0$, satisfy (52) and let $\psi^{2} G\left(\alpha^{2} \psi^{2}, x\right)$ be a nondecreasing function of $x$ for every $\alpha>0$. Assume also that there exist positive continuous functions $\varphi_{1}, \varphi_{2}$ and $\varphi_{3}$ on $(0, \infty)$ satisfying (70), (71) and

$$
\psi^{2}(x)\left\{G\left(\varphi_{2}^{2}(x), x\right)-p(x) \varphi_{2}^{2}(x)\right\}<a .
$$

Then (1) has a nontrivial nonoscillatory solution y such that $\Sigma$, defined by (13) with $\omega(x) \equiv 1$, satisfies (72) for $0<x<\infty$, and that

$$
\varphi_{3}(x)<y(x)<\varphi_{1}(x) .
$$

It will be convenient first to prove the following.

LEMMA 7. Let $\psi$ and $G$ satisfy the conditions of Theorem 5. If $y$ is a positive local solution of (1) defined near $x_{0}$, and if the energy function for $y$, with $\omega \equiv 1$, satisfies $\Sigma\left(x_{0}\right)<0$, then $y$, or any left continuation of $y$, can be continued to 0 , and will be positive there. 
Proof. As shown in $\S 5$, the conditions on $\psi$ and $G$ imply that $\Sigma$ is nondecreasing, thus it is negative to the left of $x_{0}$, on the interval of definition of $y$. The same is clearly true for the energy function for any left continuation of $y$. Since $\Sigma$ must be positive at a zero of $y$, it follows that $y$, or any left continuation of $y$, is positive to the left of $x_{0}$. Therefore $y$ or any left continuation of $y$ can be continued to 0 .

Proof of Theorem 5. For $n=1,2,3, \ldots$, let $y_{n}$ denote a solution of (1) determined by initial conditions $y_{n}(n)>0, y_{n}^{\prime}(n)$ which make $\Sigma_{n}(x)=\Sigma\left(x, y_{n}(x)\right) \leqq a$ at $x=n$. There do exist such initial conditions because of (73). Because of Lemma 7, $y_{n}$ can be assumed to be defined on $(0, n]$ and will be positive there; because it is nondecreasing $\Sigma=\Sigma_{n}$ will satisfy (72) for $0<x<n$. By virtue of (70), (71) and (72), $y=y_{n}$ must satisfy (74) for $0<x<n$. On every compact subinterval of $(0, \infty)$, $\left\{y_{n}(t)\right\}$ is a bounded sequence in view of (74). By (13) and (72) with $\omega \equiv 1, y=y_{n}$, and $\Sigma=\Sigma_{n}$, we conclude that $\left\{y_{n}^{\prime}(t)\right\}$ is also uniformly bounded on every compact subinterval. It is thus possible to choose a subsequence $\left\{y_{n_{k}}\right\}$ which is convergent on compact subintervals of $(0, \infty)$ to a solution $y$ of $(1)$ and such that $\left\{y_{n_{k}}^{\prime}\right\}$ converges uniformly on compact intervals to $y^{\prime}$. It follows that $\varphi_{3}(x) \leqq y(x) \leqq \varphi_{1}(x)$, $0<x<\infty$, and from (13) that (71) holds for the energy function for $y$, for $0<x<\infty$. In view of (70) and (71) the above estimate on $y$ can be strengthened to (74) for $0<x<\infty$. This completes the proof of Theorem 5 .

REMARK 3. We note that condition (71) is somewhat trivial in that the existence of the function $\varphi_{3}$ is always guaranteed, e.g., we may take

$$
\varphi_{3}(x)=\left((-a)^{1 / 2} / 2\right) \psi^{-1}(x) p^{-1 / 2}(x) .
$$

Corollary 11. Let

$$
|\beta|<(\gamma-1) / 2
$$

and let $q(x) x^{(\gamma+3) / 2-\beta}$ be a nondecreasing function of $x$. If

$$
\lim _{x \rightarrow \infty} q(x) x^{(\gamma+3) / 2-\beta}<\infty
$$

then (2) has a solution $y(x)$ positive on $(0, \infty)$ and such that

$$
0<\liminf _{x \rightarrow \infty} x^{-(1-\varepsilon) / 2} y(x) \leqq \limsup _{x \rightarrow \infty} x^{-(1-\varepsilon) / 2} y(x)<\infty,
$$

where $\varepsilon=2 \beta(\gamma-1)^{-1}$.

Compare the hypothesis here with the hypothesis of the oscillation criteria of Jasný [7] and Kurzweil [9] (or see Corollary 10).

Proof. Take $\psi=x^{(1+\varepsilon) / 2}$ so that $\psi$ satisfies (11) with $p(x)=\left(1-\varepsilon^{2}\right) / 4 x^{2}$. For appropriate values of $a<0, \mu_{1}, \mu_{2}, \mu_{3}>0$, the conditions (70), (71) and (73) will be satisfied for

$$
\mu_{1}^{-1} \varphi_{1}(x)=\mu_{2}^{-1} \varphi_{2}(x)=\mu_{3}^{-1} \varphi_{3}(x)=x^{(1-\varepsilon) / 2} .
$$


REMARK 4. The conditions (75) and (76) imply that $x q(x) \leqq k x^{\sigma}$, where $\sigma=\beta-(\gamma+1) / 2<-1$. Thus $q(x)$ satisfies (as it must) the integral condition of Atkinson [1], for the existence of nonoscillatory solutions of (2) mentioned earlier.

EXAMPLE. Consider the Emden-Fowler equation

$$
y^{\prime \prime}+x^{v}|y|^{\gamma} \operatorname{sgn} y=0, \quad x>0,
$$

with $-2>\nu>-(1+\gamma), \gamma>1$. Then $q(x)=x^{\nu}$ will satisfy the conditions of Corollary 11 with $\beta=\nu+(\gamma+3) / 2$. If one takes $\varepsilon=2 \beta /(\gamma-1)=1+2(\nu+2) /(\gamma-1)$, one finds that (77) has a solution $y$ given by

$$
y(x)=\left[\left(1-\varepsilon^{2}\right) / 4\right]^{1 /(y-1)} x^{(1-\varepsilon) / 2} .
$$

Atkinson's theorem yields the existence of bounded nonoscillatory solutions, the so-called Emden solutions of (77), see [5]. An Emden solution of (77) is uniquely determined by its value at $\infty$, in particular, there is a unique solution $y_{0}$ of (77) with $y_{0}(\infty)=1$. The Emden solution $y$ of (77) with $y(\infty)=c>0$ is given by $y(x)=a^{-(1-\varepsilon) / 2} y_{0}(a x)$ where $a^{-(1-\varepsilon) / 2}=c$. Finally, if $-2>v>-(\gamma+3) / 2$, then any solution of (77) which is not at least to within a factor of -1 an Emden solution or the solution given by (78) is oscillatory; see [5]. It follows from Atkinson's theorem that (77) has only oscillatory solutions, excepting the trivial one, when $\nu \geqq-2$, and it will follow from $\S 7$ that $(77)$ is nonoscillatory when $\nu \leqq-(\gamma+3) / 2$. These results are also to be found in [5].

REMARK 5. Complementary results to Corollary 11 for the sublinear equation (2), $0<\gamma<1$, can be found in Belohorec [2]. We leave the formulation of the corresponding generalization for the sublinear equation (1) to the reader.

7. Nonoscillation theorem for the superlinear equation. We prove in this section the counterpart of Theorem 4, namely a nonoscillation theorem for the superlinear equation. We assume in this section that $F$ satisfies (9) and as before that (11), with $p$ positive and continuous on $(0, \infty)$, is nonoscillatory.

THEOREM 6. Let $\varphi$ be a positive solution of $(11)$, let $\psi \in C^{3}(0, \infty)$ and suppose that

(79) $\psi>0,\left(\psi^{2}\right)^{m} \leqq 0,0<x<\infty$,

(80) $\varphi(x)=o(\psi(x))$, as $x \rightarrow \infty$,

(81) $\lim _{\inf _{x \rightarrow \infty}} \varphi^{2}\left(\psi^{2}\right)^{\prime \prime}(x) \geqq c_{0}>0$,

(82) $\lim \inf _{x \rightarrow \infty}-\varphi^{3} \varphi^{\prime \prime}(x) \geqq c_{1}>0$.

Suppose in addition that $\psi^{2} G\left(\alpha^{2} \psi^{2}, x\right)$ is a nonincreasing function of $x$ for every $\alpha>0$. Then any solution $y$ of (1) satisfying

(83) $\int^{\infty}\left|y\left(\varphi^{2}\right)^{\prime \prime}\right| d x<\infty$

must be nonoscillatory.

Proof. The theorem will follow readily if we can show that for any oscillatory solution $y(x)$ for which (83) holds, there must exist a constant $B_{0}$ such that

(84) $y^{2}(x) \leqq B_{0} \varphi^{2}(x)$. 
Indeed suppose that $y(x)$ is an oscillatory solution of (1) satisfying (84). Then since $F$ is positive and satisfies (9), we have for any $\alpha>0$

$$
G\left(\alpha^{2} \psi^{2}, x\right) \geqq c\left(\alpha^{2} \psi^{2}-B_{0} \varphi^{2}\right) F\left(B_{0} \varphi^{2}, x\right) .
$$

Thus because of (80), there exists a positive constant $K_{0}>0$ such that

(85) $G\left(\alpha^{2} \psi^{2}, x\right) \geqq K_{0} \psi^{2} F\left(B_{0} \varphi^{2}, x\right)$,

for all sufficiently large $x$. Now for any solution $y(x)$ of (1) satisfying (84), we have, on account of (9) and (85),

$$
F\left(y^{2}(x), x\right) \leqq c^{-1} F\left(B_{0} \varphi^{2}(x), x\right) \leqq\left(c K_{0}\right)^{-1} \psi^{-2}(x) G\left(\alpha^{2} \psi^{2}(x), x\right) .
$$

By hypothesis, the function $\psi^{2} G\left(\alpha^{2} \psi^{2}, x\right)$ is nonincreasing, thus we have, in view of the above inequalities,

(86) $F\left(y^{2}(x), x\right) \leqq K_{1}(\psi(x))^{-4}$

for some positive constant $K_{1}$ and all sufficiently large $x$. From (80) and (82), we also have, for sufficiently large $x$,

$$
\begin{aligned}
(\psi(x))^{-4} & \leqq(\psi(x))^{-1}(\varphi(x))^{-3} \leqq-\left(2 / c_{1}\right) \varphi^{\prime \prime}(x)(\psi(x))^{-1} \\
& =\left(2 / c_{1}\right)(\varphi(x) / \psi(x)) p(x) .
\end{aligned}
$$

Using (87) in (86), we have, for some appropriate constant $K_{2}>0$,

$$
F\left(y^{2}(x), x\right) \leqq K_{2}(\varphi(x) / \psi(x)) p(x) .
$$

Since $\varphi(x) / \psi(x)=o(1)$ as $x \rightarrow \infty$, we can pick $x_{0}$ sufficiently large so that, for $x \geqq x_{0}, F\left(y^{2}(x), x\right) \leqq p(x)$. The hypothesis that equation (11) is nonoscillatory together with Sturm's comparison theorem now implies that $y(x)$ must be nonoscillatory, thus contradicting our assumption.

It remains to show that an oscillatory solution of (1) for which (83) holds also satisfies (84) for some $B_{0}<\infty$. To this end, we first prove the following.

LeMma 8. Let $y$ be an oscillatory solution of (1) satisfying (83). Then there exists $a$ constant $B_{0}$ such that if $y(a)=y^{\prime}(b)=0$ and $y(x) \neq 0$ for $a<x<b$, then

$$
\int_{a}^{b}\left[y^{\prime 2}(x) /\left(\varphi^{2}\right)^{\prime}(x)\right] d x \leqq B_{0} .
$$

Proof. It follows from Lemma 1 and (79) that for any solution $y(x)$ of (1), $\Sigma(x)=\Sigma(x, y(x))$ (with $\omega(x) \equiv 1)$ is a nonincreasing function of $x$, and in fact we have from (17) that when $F$ is of class $C^{1}$,

$$
(d / d x) \Sigma(x) \leqq \frac{1}{2}\left(\psi^{2}\right)^{m} y^{2} .
$$

Integrating (90) from $a$ to $b$, we have

$$
\psi^{\prime 2} y^{2}(b)+\psi \psi^{\prime \prime} y^{2}(b)+\psi^{2} G\left(y^{2}, b\right)-\psi^{2} y^{\prime 2}(a) \leqq \frac{1}{2} \int_{a}^{b}\left(\psi^{2}\right)^{m} y^{2}(x) d x,
$$

and by the usual argument, this inequality remains valid even if $F$ is not $C^{1}$. 
On the other hand, we observe that

$$
\begin{aligned}
\frac{1}{2} \int_{a}^{b}\left(\psi^{2}\right)^{m} y^{2}(x) d x & =\left.\frac{1}{2} y^{2}\left(\psi^{2}\right)^{\prime \prime}(x)\right|_{a} ^{b}-\int_{a}^{b}\left(\psi^{2}\right)^{\prime \prime} y y^{\prime}(x) d x \\
& =\frac{1}{2}\left(\psi^{2}\right)^{\prime \prime} y^{2}(b)-\int_{a}^{b}\left(\psi^{2}\right)^{\prime \prime} y y^{\prime}(x) d x
\end{aligned}
$$

Combining (91) and (92), we obtain

$$
\int_{a}^{b}\left(\psi^{2}\right)^{\prime \prime} y y^{\prime}(x) d x \leqq \psi^{2} y^{\prime 2}(a)-\psi^{2} G\left(y^{2}(b), b\right) \leqq \psi^{2} y^{\prime 2}(a),
$$

and since the monotonicity of $\Sigma$ implies that $\psi^{2} y^{\prime 2}$ decreases from one zero to another zero of $y$ to the right, the integral on the right is bounded independently of the interval $[a, b]$, i.e.

$$
\int_{a}^{b}\left(\psi^{2}\right)^{\prime \prime} y y^{\prime}(x) d x \leqq B
$$

where $B$ is a constant independent of $[a, b]$. Next we note that if we assume $y(x)>0$ for $a<x<b$, then on that interval

$$
\begin{aligned}
\left(\varphi^{2} y^{\prime}-y\left(\varphi^{2}\right)^{\prime}\right)^{\prime} & =\varphi^{2} y^{\prime \prime}-y\left(\varphi^{2}\right)^{\prime \prime}=-\varphi^{2} y F\left(y^{2}, x\right)-y\left(\varphi^{2}\right)^{\prime \prime} \\
& \leqq-y\left(\varphi^{2}\right)^{\prime \prime} .
\end{aligned}
$$

Integrating the above estimate and using (83) we obtain

$$
\varphi^{2} y^{\prime} \leqq y\left(\varphi^{2}\right)^{\prime}+C_{0}
$$

where $C_{0}$ is an appropriate constant again independent of the interval $[a, b]$. This independence follows since the function $\left(\varphi^{2} y^{\prime}-y\left(\varphi^{2}\right)^{\prime}\right)(x)$ must vanish at some point of $(a, b)$. Next we use (94) to obtain the following estimate:

$$
\begin{aligned}
\int_{b}^{a} \frac{y^{\prime 2}}{\left(\varphi^{2}\right)^{\prime}} d x & =\int_{a}^{b} \frac{y^{\prime}}{\varphi^{2}\left(\varphi^{2}\right)^{\prime}} \varphi^{2} y^{\prime} d x \\
& \leqq \int_{a}^{b} \frac{y^{\prime}}{\varphi^{2}\left(\varphi^{2}\right)^{\prime}}\left(y\left(\varphi^{2}\right)^{\prime}+C_{0}\right) d x \\
& \leqq \int_{a}^{b} \frac{y y^{\prime}}{\varphi^{2}} d x+C_{0} \int_{a}^{b} \frac{y^{\prime}}{\varphi^{2}\left(\varphi^{2}\right)^{\prime}} d x
\end{aligned}
$$

Using (81) and Schwarz's inequality, we obtain, provided $a$ is sufficiently large,

$$
\int_{a}^{b} \frac{y^{\prime 2}}{\left(\varphi^{2}\right)^{\prime}} d x \leqq \frac{2}{c_{0}} \int_{a}^{b}\left(\psi^{2}\right)^{\prime \prime} y y^{\prime} d x+C_{0}\left(\int_{a}^{b} \frac{y^{\prime 2}}{\left(\varphi^{2}\right)^{\prime}} d x\right)^{1 / 2}\left(\int_{a}^{b} \frac{d x}{\varphi^{4}\left(\varphi^{2}\right)^{\prime}}\right)^{1 / 2}
$$

from (82), we have, for large $x$,

so that

$$
\varphi^{\prime}(x) \geqq \frac{c_{1}}{2} \int_{x}^{\infty} \frac{d t}{\varphi^{3}(t)}
$$

$$
\left(\varphi^{2}\right)^{\prime}(x) \geqq c_{1} \varphi(x) \int_{x}^{\infty} \frac{d t}{\varphi^{3}(t)}
$$


Now we use (96) to estimate the last integral in (95) as follows:

$$
\int_{a}^{b} \frac{d x}{\varphi^{4}\left(\varphi^{2}\right)^{\prime}} \leqq \frac{1}{c_{1}} \int_{a}^{b}\left(d x / \varphi^{5} \int_{x}^{\infty} \frac{d t}{\varphi^{3}(t)}\right) \leqq \frac{2}{c_{1} \lambda_{0}} \int_{a}^{b} \frac{d x}{\varphi^{3}(x)}
$$

where

$$
\frac{1}{\lambda_{0}}=\limsup _{x \rightarrow \infty}\left(\varphi^{2} \int_{x}^{\infty} \frac{d t}{\varphi^{3}(t)}\right)^{-1}=\limsup _{x \rightarrow \infty} \frac{-\varphi^{\prime} / \varphi^{3}}{-1 / \varphi^{3}} \leqq \varphi^{\prime}(a)
$$

Denote by $\mu$ the integral $\int_{a}^{b} y^{\prime 2} /\left(\varphi^{2}\right)^{\prime} d x$. Substituting (97) into (95), and using (93) we obtain

$$
\mu \leqq B_{1}+B_{2} \mu^{1 / 2},
$$

where $B_{1}, B_{2}$ are constants. It then follows from (98) that $\mu \leqq B_{0}$ for some appropriate constant $B_{0}$. This completes the proof.

Now let $a$ and $b$ be as in the above lemma. For $a \leqq x \leqq b$, by Schwarz's inequality

$$
|y(x)| \leqq \int_{a}^{x}\left|y^{\prime}(t)\right| d t \leqq\left(\int_{a}^{b} \frac{y^{\prime 2}}{\left(\varphi^{2}\right)^{\prime}} d t\right)^{1 / 2}\left(\int_{a}^{x}\left(\varphi^{2}\right)^{\prime} d t\right)^{1 / 2}
$$

So, by (89), $y^{2}(x) \leqq B_{0} \varphi^{2}(x)$, for $a \leqq x \leqq b$. Then for any oscillatory solution $y(x)$, it follows from the concavity of $y$ between zeros and the fact that $\varphi$ is a solution of (11) that (84) holds for all large $x$. This completes the proof of the theorem.

REMARK 6. Clearly condition (4) implies (9) for the function $F$. Also, by taking $\varphi(x)=x^{1 / 2}$ and $\psi(x)=(x \log x)^{1 / 2}$ in Theorem 6 , we obtain Theorem 2 of [3] which is a generalization of earlier results of Kiguradze [8] and Nehari [13]. Note that in this case, since $\left(\varphi^{2}\right)^{\prime \prime} \equiv 0$, condition (83) is trivially satisfied.

As an example of a function $F(t, x)$ satisfying (9) but not (4), we may take $F(t, x)=q(x) \exp (\xi(t)-\eta(t))$, where $q(x) \geqq 0, \xi(t)$ is nondecreasing and $\eta(t)$ is uniformly bounded for all $t$.

\section{REFERENCES}

1. F. V. Atkinson, On second-order non-linear oscillations, Pacific J. Math. 5 (1955), 643-647. MR 17, 264.

2. S. Belohorec, On some properties of the equation $y^{\prime \prime}(x)+f(x) y^{\alpha}(x)=0,0<\alpha<1$, Mat. Casopis Sloven. Akad. Vied 17 (1967), 10-19. MR 35 \#5703.

3. C. V. Coffman and J. S. W. Wong, On a second order nonlinear oscillation problem, Trans. Amer. Math. Soc. 147 (1970), 357-366. MR 41 \#2123.

4. - Second order nonlinear oscillations, Bull. Amer. Math. Soc. 75 (1969), 1379-1382. MR 40 \#449.

5. R. H. Fowler, Further studies of Emden's and similar differential equations, Quart. J. Math. 2 (1931), 259-288.

6. J. W. Heidel, A nonoscillation theorem for a nonlinear second order differential equation, Proc. Amer. Math. Soc. 22 (1969), 485-488. MR 40 \#1648.

7. M. Jasný, On the existence of an oscillating solution of the nonlinear differential equation of the second order $y^{\prime \prime}+f(x) y^{2 n-1}=0, f(x)>0$, Časopis Pèst. Mat. 85 (1960), 78-83. (Russiañ) MR 26 \#408. 
8. I. T. Kiguradze, On the conditions for oscillation of solutions of the differential equation $u^{\prime \prime}+a(t)|u|^{n} \operatorname{sgn} u=0$, Ćasopis Pěst. Mat. 87 (1962), 492-495. (Russian) MR 31 \#6026.

9. J. Kurzweil, $A$ note on oscillatory solution of equation $y^{\prime \prime}+f(x) y^{2 n-1}=0$, Casopis Pěst. Mat. 85 (1960), 357-358. (Russian) MR 23 \#A3322.

10. J. W. Macki and J. S. W. Wong, Oscillation of solutions to second-order nonlinear differential equations, Pacific J. Math. 24 (1968), 111-117. MR 37 \#507.

11. Z. Nehari, On a class of nonlinear second-order differential equations, Trans. Amer. Math. Soc. 95 (1960), 101-123. MR 22 \#2756.

12. - Characteristic values associated with a class of nonlinear second-order differential equations, Acta Math. 105 (1961), 141-175. MR 23 \#A1097.

13. — A nonlinear oscillation problem, J. Differential Equations 5 (1969), 452-460. MR 38 \#3514.

14. J. S. W. Wong, Some properties of solutions of $u^{\prime \prime}(t)+a(t) f(u) g\left(u^{\prime}\right)=0$. III, SIAM J. Appl. Math. 14 (1966), 209-214. MR 34 \#3020.

15. ——, On second order nonlinear oscillation, Funkcial. Ekvac. 11 (1969), 207-234. MR 39 \#7221.

Appendix: continuation and local uniqueness of solutions of generalized EmdenFowler equations. We are concerned with establishing continuability and local uniqueness of solutions of the following second order ordinary differential equation:

$$
y^{\prime \prime}+y F\left(y^{2}, x\right)=0, \quad x>0,
$$

where $y F\left(y^{2}, x\right)$ is continuous for $x>0$ and $|y|<\infty$, and $F(t, x)$ is nonnegative for $x>0$ and $t>0$. The prototype of equation (1), namely

$$
y^{\prime \prime}+q(x)|y|^{\gamma} \operatorname{sgn} y=0, \quad x>0,
$$

where $q(x)$ is continuous and nonnegative, has received considerable attention in recent years. We classify equation (1) as superlinear or sublinear according to whether $F(t, x)$ is monotone increasing or decreasing in $t$. More precisely, we say that equation (1) is superlinear if

$$
F\left(t_{2}, x\right) \geqq F\left(t_{1}, x\right), \quad t_{2} \geqq t_{1},
$$

and sublinear if

$$
F\left(t_{2}, x\right) \leqq F\left(t_{1}, x\right), \quad t_{2} \geqq t_{1} .
$$

When these conditions are specialized to equation (2), we obtain $\gamma \geqq 1$ for superlinearity and $\gamma \leqq 1$ for sublinearity.

In case of equation (2) when $\gamma \geqq 1$, the local uniqueness of the zero solution, i.e. that a solution $y(x)$ satisfying the initial conditions

$$
y\left(x_{0}\right)=y^{\prime}\left(x_{0}\right)=0
$$

for some $x_{0}$ must be the identically zero solution, is well known. It is also not difficult to see that the same remains valid for the more general equation (1) 
subject to condition (3). This was observed by Nehari [12]; for completeness we include a slightly simplified version of his argument here. Suppose that $y(x)$ is a solution of (1) satisfying (5). Choose $\varepsilon>0$ such that

$$
\int_{x_{0}}^{x_{0}+\varepsilon}\left(x_{0}+\varepsilon-s\right) F(1, s) d s<1
$$

which is always possible since $F(t, x)$ is continuous in its variables. Integrating (1) from $x_{0}$ to $x, x \in\left[x_{0}, x_{0}+\varepsilon\right]$, we obtain

$$
y(x)=\int_{x_{0}}^{x}(x-s) y(x) F\left(y^{2}(x), s\right) d s .
$$

We may also assume that $\varepsilon>0$ is sufficiently small so that

$$
\sup _{x_{0} \leqq x \leqq x_{0}+\varepsilon}|y(x)| \leqq c<1 .
$$

Since $y(x)$ is continuous, there exists $x_{1} \in\left[x_{0}, x_{0}+\varepsilon\right]$ at which $\left|y\left(x_{1}\right)\right|=c$. Note that equation (1) is symmetric with respect to $y$, that is if $y(x)$ is a solution of (1), so is $-y(x)$. Therefore we may assume $y\left(x_{1}\right)=c$. Evaluating (7) at $x_{1}$, we have by (6)

$$
\begin{aligned}
c & =\int_{x_{0}}^{x_{1}}\left(x_{1}-s\right) y(x) F\left(y^{2}(s), s\right) d s \\
& \leqq c \int_{x_{0}}^{x_{1}}\left(x_{1}-s\right) F\left(c^{2}, x\right) d s \\
& \leqq c \int_{x_{0}}^{x_{0}+\varepsilon}\left(x_{0}+\varepsilon-s\right) F\left(c^{2}, s\right) d s<c,
\end{aligned}
$$

which is a contradiction. Thus $y(x) \equiv 0$. A further analysis of the above proof shows that in the superlinear case the uniqueness of the zero solution in fact follows from the corresponding property for the linear equation. Indeed every solution $y(x)$ of (1) satisfies the linear equation $y^{\prime \prime}+p(x) y=0$, where $p(x)$ $=F\left(y^{2}(x), x\right)$ is continuous, which clearly has the required uniqueness property. The solution of an arbitrary initial value problem for equation (1) is of course unique when $F(t, x)$ satisfies a locally uniform Lipschitz condition in $t$. This is the case in particular for equation (2) when $\gamma \geqq 1$.

We make note next of the fact that, for the sublinear case of (1), any solutions defined locally can be extended to $(0, \infty)$. Proofs for the sublinear case of $(2)$ have been given by Heidel, [A-5], and Belohorec [2]. As in [A-5], we may derive a general result for equation (1) from a theorem of Wintner, see Hartman [A-4, p. 29]. Indeed, the equation (1) is equivalent to the vector equation $\eta^{\prime}=f(x, \eta)$, where $\eta(x)=\left(y^{\prime}(x), y(x)\right), f(x, \eta)=\left(-y F\left(y^{2}, x\right), y^{\prime}\right)$. If we use the vector norm $|\eta|$ $=\max \left(\left|\eta_{1}\right|,\left|\eta_{2}\right|\right)$ then (4) implies that $|f(x, \eta)| \leqq(1+K(x))|\eta|$, where $K(x)$ $=\max _{0<\xi \leqq x}\left(\max _{0 \leqq y \leqq 1} y F\left(y^{2}, \xi\right)\right)$. The scalar equation $r^{\prime}=(1+K(x)) r$ has all of its solutions defined on $(0, \infty)$; thus by the result of Wintner just referred, any locally defined solution of the sublinear equation (1) can be extended to $(0, \infty)$. 
Of course a weaker condition than (4) will suffice in order that all solutions of (1) be continuable. One such useful condition is the domination of $F(t, x)$, for large $t$, by a function independent of $t$. We formulate this more precisely as follows. If there exists a continuous nonnegative function $p(x)$, and if for every compact interval $[a, b]$ in $(0, \infty)$, there exists an $M>0$ such that $F(t, x) \leqq p(x)$ for $t \geqq M$ and $a \leqq x \leqq b$, then any locally defined solution of (1) can be extended to $(0, \infty)$. The proof is similar to that given in the preceding paragraph.

In view of the above remarks it is apparent that the more subtle questions are those of continuability of solutions of the superlinear equation and of the uniqueness of the zero solution of the sublinear equation. For the first order scalar equation, althou ${ }^{\prime}$ h the problem there is not so deep, an analogous situation occurs as is illustrated by the simple equation $y^{\prime}=|y|^{\gamma} \operatorname{sgn} y$ considered for $0<\gamma<1$ and $1<\gamma<\infty$. There is, for this first order equation, a sort of "duality" between the nonuniqueness of the zero solution, for $0<\gamma<1$, and the failure of global existence of solutions when $1<\gamma<\infty$. As we shall see, a more striking and more interesting duality exists in the second order case between the two problems mentioned at the beginning of this paragraph. (Cf. Heidel [A-5] concerning equation (2).)

We consider first the problem of continuability of solutions of the superlinear equation. For the special case of equation (2), this problem has been considered by Hastings [A-3], Coffman and Ullrich [A-1], Heidel [A-5] and in related papers, Jasný [7], Kiguradze [8], Moore and Nehari [A-8], Willett and Wong [A-10]. A systematic study does not seem to have been made concerning the continuability problem for equation (1). Some general results concerning equations more general than (1) may be found in Hastings [A-3], but they seem to be not particularly useful in analyzing equation (1). Results generalizing that of Coffman and Ullrich [A-1] for the Emden-Fowler equation (2) are also given in Ullrich [A-9]. Similar remarks concerning the continuability of solutions may be found in Nehari [11], [13], Coffman and Wong [3].

Let $0 \leqq a<b \leqq \infty$. By a local solution of $(1)$ in $(a, b)$, we shall mean a solution of (1) defined on a nonempty open subinterval of $(a, b)$. By the phrase, "all solutions of (1) are continuable on $(a, b)$," we shall mean that every local solution in $(a, b)$ of (1) has a $C^{2}$ extension on $(a, b)$. If $b$ is finite we shall say that all solutions of (1) are continuable through $b$ if for some $a<b$ every local solution of (1) in $(a, b)$ has a $C^{2}$ extension on an open interval containing $b$.

The main result of [A-1] states that if $q(x)$ is positive and locally of bounded variation on $(a, b)$ then all solutions of $(2)$ are continuable on $(a, b)$. The same argument as the one used in the proof of the result just quoted gives the following: if $b<\infty$ and if $q(x)$ is positive on $(a, b)$ and $\log q(x)$ has finite upper variation on $(a, b)$, then all solutions of (1) are continuable through $b$. This last result is of interest primarily as a criterion of continuability of solutions of (2) through an isolated zero of $q(x)$. Another result along this line is due to Heidel [A-5], and states that if $\left[(b-x)^{\alpha+3} q(x)\right]^{\prime}$ exists and is nonpositive on some left neighborhood of $b$, then all 
solutions of (2) are continuable through $b$. Actually Heidel [A-5] stated this result in a somewhat different form, and he has explicitly assumed that $q(b)=0$ there, which is an unnecessary assumption.

Concerning equation (1), when (3) holds, Ullrich [A-9] has given the following generalization of the continuability result of [A-1] for (2): if there exists a nondecreasing function $h(x)$ on $(a, b)$ such that, for all $t>0$,

$$
\left|\log F\left(t, x_{2}\right)-\log F\left(t, x_{1}\right)\right| \leqq h\left(x_{2}\right)-h\left(x_{1}\right), \quad a \leqq x_{1}<x_{2} \leqq b,
$$

then all solutions of (1) are continuable on $(a, b)$. Let

$$
G(t, x)=\int_{0}^{t} F(s, x) d s
$$

Inspection of Ullrich's proof shows that (8) can be replaced by

$$
\left|\log G\left(t, x_{2}\right)-\log G\left(t, x_{1}\right)\right| \leqq h\left(x_{2}\right)-h\left(x_{1}\right), \quad a \leqq x_{1}<x_{2}<b,
$$

which is implied by (8). We remark also that Ullrich's proof can be made to yield a one-sided (i.e. right) continuation criteria, namely

$$
\log G\left(t, x_{2}\right)-\log G\left(t, x_{1}\right) \leqq h\left(x_{2}\right)-h\left(x_{1}\right), \quad a \leqq x_{1}<x_{2}<b .
$$

For equation (2), where $G(t, x)=(\gamma+1)^{-1} q(x) t^{\gamma+1},(11)$ is weaker than (10) only if $q(b)=0$, however, in general, (11) is weaker than (10) even if $b<\infty$ and $G(t, x)$ is positive for $a \leqq x \leqq b, t>0$.

In Theorem A.1 below we state continuability criteria for solutions of (1) which contain all of the results quoted above. We first give a necessary condition that a solution of (1) cannot be continued through $b$ in the following lemma.

Lemma A.1. Let $y(x)$ be a solution of (1) with F satisfying (3) on $[a, b)$. Suppose that $y(x)$ cannot be continued through $b$; then $y(x)$ has infinitely many zeros in every left neighborhood of $b$ and

$$
\limsup _{x \rightarrow b-}|y(x)|=\limsup _{x \rightarrow b-}\left|y^{\prime}(x)\right|=\infty .
$$

Proof. We first show that if $y(x)$ cannot be continued through $b$ then $y(x)$ must have infinitely many zeros in every left neighborhood of $b$. Otherwise, there exists $\varepsilon>0$ such that $y(x)$ is concave toward the axis for $b-\varepsilon<x<b$, and consequently $\lim _{x \rightarrow b-} y^{\prime}(x)$ and $\lim _{x \rightarrow b-} y(x)$ exist and are finite, proving continuability.

Now suppose that lim $\sup _{x \rightarrow b-}|y(x)|<\infty$, then an integration of the differential equation yields the existence and finiteness of $\lim _{x \rightarrow b-} y(x)$ and of $\lim _{x \rightarrow b-} y^{\prime}(x)$, from which follows continuability. If lim $\sup _{x \rightarrow b-}\left|y^{\prime}(x)\right|<\infty$, then an integration yields lim $\sup _{x \rightarrow b-}|y(x)|<\infty$, and the result follows as above. 
THEOREM A.1. Let there exist a bounded nondecreasing function $h(x)$ on $(a, b)$ such that for every $\alpha>0$

$$
\begin{array}{r}
\log \left[\left(b-x_{2}\right)^{2} G\left(\alpha\left(b-x_{2}\right)^{2}, x_{2}\right)\right]-\log \left[\left(b-x_{1}\right)^{2} G\left(\alpha\left(b-x_{1}\right)^{2}, x_{1}\right)\right] \\
\leqq h\left(x_{2}\right)-h\left(x_{1}\right), \quad a<x_{1}<x_{2}<b ;
\end{array}
$$

then every local solution in $(a, b)$ of (1) possesses a continuation through $b$.

Proof of Theorem A.1. It suffices to assume that there exists a solution $y$ of (1) defined on some open interval $[\alpha, \beta), a \leqq \alpha<\beta \leqq b$, and which possesses no proper right extension and to show that this assumption leads to a contradiction. In view of Lemma A.1 it suffices to show that the given solution $y$ is bounded on $[\alpha, \beta)$. The existence of a proper right continuation, and thus the contradiction, follows.

Put

$$
\Phi(x)=\left(y(x)+(b-x) y^{\prime}(x)\right)^{2}+(b-x)^{2} G\left(y^{2}(x), x\right) ;
$$

then if $G$ is of class $C^{1}$ we have

$$
\begin{aligned}
\Phi^{\prime}(x)= & 2\left(y(x)+(b-x) y^{\prime}(x)\right)(b-x) y^{\prime \prime} \\
& +\left(\frac{y^{\prime}}{b-x}+\frac{y}{(b-x)^{2}}\right) \frac{\partial}{\partial \alpha}\left[(b-x)^{2} G\left(\alpha^{2}(b-x)^{2}, x\right)\right]_{\alpha=(y /(b-x))} \\
& +\frac{\partial}{\partial x}\left[(b-x)^{2} G\left(\alpha^{2}(b-x)^{2}, x\right)\right]_{\alpha=(y /(b-x))} \\
= & 2(b-x)\left(y(x)+(b-x) y^{\prime}\right)\left(y^{\prime \prime}+y F\left(y^{2}, x\right)\right) \\
& +\frac{\partial}{\partial x}\left[(b-x)^{2} G\left(\alpha^{2}(b-x)^{2}, x\right)\right]_{\alpha=(y /(b-x)) .}
\end{aligned}
$$

For $G$ of class $C^{1},(12)$ implies

$$
\frac{\partial}{\partial x}\left[(b-x)^{2} G\left(\alpha^{2}(b-x)^{2}, x\right)\right] \leqq h^{\prime}(x)\left[(b-x)^{2} G\left(\alpha^{2}(b-x)^{2}, x\right)\right],
$$

and thus

$$
\Phi^{\prime}(x) \leqq 2(b-x)\left(y(x)+(b-x) y^{\prime}\right)\left(y^{\prime \prime}+y F\left(y^{2}, x\right)\right)+h^{\prime}(x) \Phi(x) .
$$

We have therefore the differential inequality $\Phi^{\prime}(x) \leqq h^{\prime}(x) \Phi(x), \alpha \leqq x<\beta$, integration of which yields

$$
\Phi(x) \leqq \Phi(\alpha) \exp [h(x)-h(\alpha)], \quad \alpha \leqq x<\beta .
$$

If $G$ is not of class $C^{1}$, choose a sequence of $C_{1}$ functions $G_{n}(t, x)$ such that, as $n \rightarrow \infty, \quad G_{n}(t, x) \rightarrow G(t, x)$ and $F_{n}(t, x)=(\partial / \partial t) G_{n}(t, x) \rightarrow F(t, x)$ uniformly on compact subsets of $a<x<b, t \geqq 0$, and so that, in addition, for all $\alpha>0, n=1,2, \ldots$,

$$
(\partial / \partial x)\left[(b-x)^{2} G_{n}\left(\alpha^{2}(b-x)^{2}, x\right)\right] \leqq h^{\prime}(x)(b-x)^{2} G_{n}\left(\alpha^{2}(b-x)^{2}, x\right) .
$$

Taking

$$
\Phi_{n}(x)=\left(y(x)+(b-x) y^{\prime}(x)\right)^{2}+(b-x)^{2} G_{n}\left(y^{2}(x), x\right),
$$


we obtain

$$
\Phi_{n}^{\prime}(x) \leqq 2(b-x)\left(y(x)+(b-x) y^{\prime}\right)\left(y^{\prime \prime}+y F_{n}\left(y^{2}, x\right)\right)+h^{\prime}(x) \Phi_{n}(x),
$$

for $\alpha \leqq x<\beta$. Thus for arbitrary $\beta^{\prime}, \alpha<\beta^{\prime}<\beta$,

$$
\Phi_{n}^{\prime}(x) \leqq C_{n}+h^{\prime}(x) \Phi_{n}(x), \quad \alpha \leqq x \leqq \beta^{\prime},
$$

where $C_{n}=\sup _{\alpha \leqq x \leqq \beta^{\prime}}\left|2(b-x)\left(y(x)+(b-x) y^{\prime}(x)\right)\left(y^{\prime \prime}+y F_{n}\left(y^{2}, x\right)\right)\right|$, so that $\lim _{n \rightarrow \infty} C_{n}=0$. Integrating the above differential inequality and letting $n \rightarrow \infty$, then since $\lim _{n \rightarrow \infty} \Phi_{n}(x)=\Phi(x), \alpha \leqq x<\beta$, we obtain $\Phi(x) \leqq \Phi(\alpha) \exp [h(x)-h(\alpha)]$, and since $\beta^{\prime}<\beta$ was arbitrary, it follows that (14) holds regardless of whether $G$ is of class $C^{1}$. Since $G(t, x)$ is positive, (11) and (12) imply that there exists a constant $K<\infty$ such that $(y(x) /(b-x))^{\prime} \leqq K(b-x)^{-2}, \alpha \leqq x<\beta$, and integration of this differential inequality shows that $y(x)$ is bounded on $(\alpha, \beta)$.

Corollary A.1. Let $\log \left[(b-x)^{\gamma+3} q(x)\right]$ have finite upper variation on $(a, b)$, then every local solution in $(a, b)$ of $(2)$ possesses a continuation through $b$.

The proof of the main theorem of [A-1], concerning continuability of solutions of (2), consists in proving boundedness of $\frac{1}{2}\left(y^{\prime}(x)\right)^{2}+(\gamma+1)^{-1} q(x)|y(x)|^{\gamma+1}$, for solutions of (2). From boundedness of this expression follows boundedness of $y^{\prime}(x)$, hence, in view of Lemma A.1, continuability of $y(x)$. An alternative approach is to consider the function

$$
((\gamma+1) / 2)\left(y^{\prime}(x)\right)^{2} / q(x)+|y(x)|^{\gamma+1},
$$

boundedness of which directly implies boundedness of $y(x)$, hence, again in view of Lemma A.1, continuability. Actually the first approach is preferable, since for right continuation this approach requires only boundedness of the upper variation of $\log q(x)$. The second approach, on the other hand, requires for right continuation the boundedness of the lower variation of $\log q(x)$, and thus this approach can never be applied to prove continuation through an isolated zero of the coefficient $q(x)$. However, the attempt to generalize the second approach leads to the following theorem, which, while it gives nothing new for equation (2), neither is it contained in Theorem A.1.

THEOREM A.2. Let there exist a bounded nondecreasing function $h(x)$ on $(a, b)$ such that for every $\alpha>0$

$$
\log F\left(\alpha, x_{2}\right)-\log F\left(\alpha, x_{1}\right) \geqq-\left(h\left(x_{2}\right)-h\left(x_{1}\right)\right), \quad a<x_{1}<x_{2}<b,
$$

then every local solution in $(a, b)$ of $(1)$ possesses a continuation through $b$.

Proof. We sketch a proof for the $C^{1}$ case. Let $G(t, x)$ be given by (9) and let $\Gamma(\omega, x)$ be defined implicitly by

$$
\Gamma(G(t, x), x)=t,
$$


then $\Gamma_{\omega}(\omega, x)=(F(\Gamma(\omega, x), x))^{-1}$, and $\Gamma_{x}(\omega, x)=-G_{x}(\Gamma(\omega, x), x) / F(\Gamma(\omega, x), x)$. If $y$ is a local solution of (1) on $(a, b)$ and if we put $\Phi(x)=\left(y^{\prime}\right)^{2}+G\left(y^{2}(x), x\right)$, then $\Phi^{\prime}(x)=G_{x}\left(y^{2}(x), x\right)$, and thus, if $\Psi(x)=\Gamma(\Phi(x), x)$, then

$$
\Psi^{\prime}(x)=\left(G_{x}\left(y^{2}(x), x\right)-G_{x}(\Psi(x), x)\right) / F(\Psi(x), x) .
$$

Since $\Phi(x) \geqq G\left(y^{2}(x), x\right)$, it follows that

$$
\Psi(x) \geqq y^{2}(x),
$$

and using (3) and (9) we obtain from (16)

$$
\Psi^{\prime}(x)=-\int_{y^{2}(x)}^{\Psi(x)} \frac{\partial}{\partial x} \log F(s, x) d s,
$$

which in view of (15) and (17) yields

$$
\Psi^{\prime}(x) \leqq h^{\prime}(x) \Psi(x) .
$$

Suppose now that $y(x)$ is a local solution of (1) defined, say, on $\left(x_{0}, x_{1}\right) \subset(a, b)$. Then integration of (18) and the use of (17) and the boundedness of $h(x)$ on $(a, b)$ lead to the conclusion that $\lim _{x \rightarrow x_{1}-}|y(x)|<\infty$. By Lemma A.1, this implies the desired result.

Yet other continuation criteria can be obtained if one is willing to impose a stronger condition than that of local bounded variation and yet weaker than the notion of differentiability of the coefficient $q(x)$ in case of equation (2). For example we can obtain continuability results by requiring the existence of upper right derivatives. The upper right derivative of a function $\Phi(x)$ is defined as

$$
D_{R}^{+} \Phi(x)=\limsup _{h \rightarrow 0^{+}} \frac{1}{h}(\Phi(x+h)-\Phi(x)) .
$$

A result in this direction is the following.

THEOREM A.3. Suppose that the function $G(t, x)$ defined in (8) is upper right differentiable with respect to $x$ for each $t$ and satisfies

$$
D_{R}^{+} G(t, x) \leqq \omega(x, G(t, x)),
$$

on $[0, X]$, where $\omega(x, r)$ is a continuous function of both variables $x$ and $r$ and nondecreasing in $r$ for each $x$. If in addition every solution of the first order equation

$$
r^{\prime}(x)=\omega(x, r(x))
$$

can be continued up to $X$, then every solution of (1) can be continued up to $X$.

Proof. We introduce the Lyapunov function for equation (1):

$$
\Phi(x)=y^{\prime 2}(x)+G\left(y^{2}(x), x\right) .
$$

Since $G(t, x)$ is right differentiable, we differentiate (21) and obtain

$$
D_{R}^{+} \Phi(x)=D_{R}^{+} G\left(y^{2}(x), x\right) \leqq \omega\left(x, G\left(y^{2}(x), x\right)\right) .
$$


By hypothesis, $\omega(x, r)$ is nondecreasing in $r$; so we have

$$
D_{R}^{+} \Phi(x) \leqq \omega(x, \Phi(x)),
$$

for all $x \in[0, X]$. An application of a differential inequality (see Hartman [A-4, p. 26]) will now give $\Phi(x) \leqq r(x), x \in[0, X]$, from which it follows that $\Phi(x)$ is bounded on $[0, X]$ and thus $y^{\prime}(x)$. The result then follows from Lemma A.1.

When restricted to equation (2) the above theorem yields the following extension of a result in Willett and Wong [A-10].

COROllaRy A.2. Let $q(x)>0$ be continuous and upper right differentiable on $[0, \infty)$, then every solution of (2) can be continued from the right on $[0, \infty)$.

Proof. Note that equation (20) in this case becomes $r^{\prime}(x)=\left(D_{R}^{+} q(x)\right) r(x)$ which has the solution $r(x)=r\left(x_{0}\right) \exp \left(\int_{x_{0}}^{x} D_{R}^{+} q(s) d s\right)$ and clearly can be continued from the right on $[0, \infty)$.

REMARK A.1. We can of course use the differential inequality technique as just given in Theorem A.3 to formulate a more general condition than (12) in order to improve Theorem A.1. The details are essentially the same and will be left to the interested reader.

We turn our attention now to the sublinear case, namely equation (1), where $F$ satisfies condition (4). Before discussing the problem of the uniqueness of the zero solution we prove several other results for this equation which are also of interest. The first two of these concern the initial value problem

$$
y\left(x_{0}\right)=0, \quad y^{\prime}\left(x_{0}\right)=a,
$$

where $a \neq 0$, and thus without loss of generality we can take $a>0$. A similar study of this problem for the superlinear equation (1), with $F$ satisfying (3) and other additional hypothesis, may be found in Moroney [A-7], and Coffman [A-2].

TheOREM A.4. Let $0<\alpha<\beta<\infty$, and let $A>0$. Then there is an $\varepsilon>0$, depending only on $F, \alpha, \beta$ and $A$ such that if $\alpha \leqq x_{0} \leqq \beta, a>A$, and $y(x)$ is a solution of (1) satisfying (23), then $y(x)>0$ for $x_{0}<x<x_{0}+\varepsilon$.

THEOREM A.5. If there exist positive constants $\delta_{1}$ and $\delta_{2}<a$, and a nonnegative function $h(x)$ defined and locally integrable on $0<\left|x-x_{0}\right|<\delta_{1}$, satisfying

$$
\int_{x_{0}}^{x_{0} \pm \delta_{1}}\left(x-x_{0}\right) h(x) d x<\infty
$$

and if

$$
\left|\omega_{2} F\left(\omega_{2}^{2}\left(x-x_{0}\right)^{2}, x\right)-\omega_{1} F\left(\omega_{1}^{2}\left(x-x_{0}^{2}\right), x\right)\right| \leqq h(x)\left|\omega_{2}-\omega_{1}\right|,
$$

for $0<\left|x-x_{0}\right|<\delta$ and $\left|\omega_{i}-a\right|<\delta_{2}, i=1,2$, then the initial value problem (23) for (1) has a locally unique solution when $a \neq 0$. 
Proof of Theorems A.4 and A.5. Let $y(x)$ be a solution of the initial value problem (23) for (1). (By remarks above $y$ can be assumed to be defined on $(0, \infty)$.) Then $\omega(x)=y(x) /\left(x-x_{0}\right)$ satisfies the integral equation

$$
\omega(x)=a-\int_{x_{0}}^{x}\left(1-\frac{\left(t-x_{0}\right)}{\left(x-x_{0}\right)}\right)\left(t-x_{0}\right) \omega(t) F\left(\left(t-x_{0}\right)^{2} \omega^{2}(t), t\right) d t .
$$

Let $\varepsilon$ be chosen so that

$$
\int_{x_{0}}^{x_{0}+\varepsilon}\left(t-x_{0}\right) F\left(\frac{1}{4} A^{2}\left(t-x_{0}\right)^{2}, t\right) d t<\frac{1}{6}
$$

then using (4) and a standard argument one can easily show that if $a>A$, then any solution $\omega$ of (26) must satisfy $\omega(x)>3 a / 4$, for $x_{0}<x<x_{0}+\varepsilon$. The assertion of Theorem A.4 clearly follows.

To prove Theorem A.5 we use the fact that local uniqueness for solutions of the initial value problem is equivalent to local uniqueness for solutions of (26). Accordingly, let $\omega_{1}$ and $\omega_{2}$ be two solutions of (26), then from (26) and (25) we have

$$
\left|\omega_{1}(x)-\omega_{2}(x)\right| \leqq\left|\int_{x_{0}}^{x}\left(t-x_{0}\right) h(t)\right| \omega_{1}(t)-\omega_{2}(t)|d t| .
$$

Using (24) and a standard argument we conclude that $\omega_{1}(x) \equiv \omega_{2}(x)$ in some neighborhood of $x_{0}$. This completes the proof of Theorem A.5.

COROllary A.3. Let $y$ be a solution of (1) and let $x_{0}$ be a cluster point of zeros of $y$. Then

$$
\lim _{x \rightarrow x_{0}} y^{\prime}(x)=\lim _{x \rightarrow x_{0}} y(x)=0 .
$$

Proof. Immediate from Theorem A.4. We remark that the above corollary also gives a simple generalization of a result of Heidel [6] for equation (2).

COROllary A.4. Let $0<\gamma<1$, then the initial value problem (23) for (2) has a locally unique solution when $a \neq 0$.

Proof. Let $0<\delta_{2}<a$, then for $\omega_{1}, \omega_{2} \geqq a-\delta_{2}$,

$$
\left(x-x_{0}\right)^{\gamma-1} q(x)\left|\left(\omega_{2}^{\gamma}-\omega_{1}^{\gamma}\right)\right| \leqq \gamma\left(x-x_{0}\right)^{\gamma-1} q(x)\left(a-\delta_{2}\right)^{\gamma-1}\left|\omega_{2}-\omega_{1}\right|,
$$

and thus the equivalent of (25) is satisfied with $h(x)=k\left(x-x_{0}\right)^{y-1} q(x)$, where $k$ is a positive constant depending only on $\delta_{2}$.

We come now to the problem of uniqueness of the zero solution for the sublinear equation (1). We make the following simple but important observation.

LEMMA A.2. If a solution $y(x)$ of (1) has

$$
y\left(x_{0}\right)=y^{\prime}\left(x_{0}\right)=0,
$$

for some $x_{0} \in(0, \infty)$, then $y$ has infinitely many zeros in every neighborhood of $x_{0}$.

Proof. If $y \equiv 0$ in a neighborhood of $x_{0}$ there is nothing to prove. We assume therefore that, for any $\varepsilon>0, y(x) \neq 0$ on the interval $x_{0}-\varepsilon<x<x_{0}$. If for some 
$\varepsilon^{\prime}, 0<\varepsilon^{\prime}<\varepsilon, y$ were of one sign, say positive, for $x_{0}-\varepsilon^{\prime}<x<x_{0}$, then since $y y^{\prime \prime} \leqq 0$, we would have $y^{\prime}(x) \geqq y^{\prime}\left(x_{0}\right)=0$ for all $x \in\left(x_{0}-\varepsilon^{\prime}, x_{0}\right)$. Consequently, $y(x) \leqq y\left(x_{0}\right)$ $=0$, which contradicts what we just assumed. This completes the proof.

We have quoted a result to the effect that when $\gamma>1$ and the upper variation of $\log q(x)$ is finite in some interval $(a, b]$ then every local solution of (2) defined in $(a, b)$ possesses a continuation through $b$. The "dual" of this result for (2) with $0<\gamma<1$ is the following: if $q(x)$ is positive and the lower variation of $\log q(x)$ is bounded in $(a, b]$, then for $x_{0} \in(a, b]$ any solution of (1) satisfying (27) vanishes identically on $\left(a, x_{0}\right)$. The latter result, like the former, is obtained from a differential inequality for the "energy function", $\frac{1}{2}\left(y^{\prime}\right)^{2}+(\gamma+1)^{-1} q(x)|y|^{\gamma+1}$. On the other hand, if $q(x)>0$ on $(a, b), q(b)=0$, and $q$ is decreasing for $b-\varepsilon<x<b$, where $\varepsilon>0$ (so that the lower variation of $\log q$ is necessarily infinite on $(a, b)$ ), then if $y$ were a solution of (2) with $0<\gamma<1$, satisfying (27), with $x_{0}=b$, and if $y$ did not vanish identically in $(b-\varepsilon, b)$ for some $\varepsilon^{\prime}>0$, then, by Lemma A.2, $y$ would have infinitely many zeros in $\left(b-\varepsilon^{\prime}, b\right)$. However the monotone character of $q(x)$ would imply that the amplitude of these oscillations is increasing in $(b-\varepsilon, b)$, which in view of (27) is impossible, thus $y$ must vanish identically in $(b-\varepsilon, b]$. A stronger result due to Heidel [A-5] states that if $(b-x)^{\gamma+3} q(x)$ is decreasing in $(b-\varepsilon, b)$ for some $\varepsilon>0$, then any solution of (2) satisfying (27), with $x_{0}=b$, vanishes identically in $(b-\varepsilon, b)$. The result in [A-5] is stated for the $C^{1}$ case and it is assumed there that $q(b)=0$. We present extensions of these results, and in fact, our results when reduced to equation (2) improve the known results mentioned above.

TheOREM A.6. Let $F$ satisfy (4) and assume that there exists a nonincreasing function $h(x)$, defined on $[a, b]$, such that, for every $\alpha>0$,

$$
\log G\left(\alpha, x_{2}\right)-\log G\left(\alpha, x_{1}\right) \geqq h\left(x_{2}\right)-h\left(x_{1}\right)
$$

for $x_{2} \geqq x_{1}$. Then for $x_{0} \in(a, b]$, any solution of (1) satisfying (27) vanishes identically in $\left(a, x_{0}\right]$.

The proof of this result will be omitted, since the arguments are similar to those used previously and those to be used below. More specifically, one shows that the function $\Phi(x)=\left(y^{\prime}\right)^{2}+G\left(y^{2}, x\right)$, when $y$ is a solution of (2) is either positive on $[a, b]$ or vanishes identically there.

The proof of Heidel's result just mentioned involves the device of throwing the singular point $b$ for (2) to infinity by means of the change of variable $y(x)$ $=(b-x) u(t), t=1 /(b-x)$. This result can be proved also by considering the "Lyapunov function"

$$
\frac{\left(y(x)+(b-x)^{\prime} y(x)\right)^{2}}{(b-x)^{\gamma+3} q(x)}+\frac{1}{\gamma+1}\left|\frac{y(x)}{b-x}\right|^{\gamma+1},
$$

for (2). The proof of Theorem A.7 below depends upon finding the appropriate generalization of the above Lyapunov function for (1). 
TheOREM A.7. Assume (1) there exist positive constants $\delta_{1}, \delta_{2}$ and there exists a positive $C^{1}$ function $\varphi(t)$ defined for $t \geqq 0$, with $\varphi(0)=0$, and $\varphi^{\prime}(t)>0$ for $t>0$, such that for $0<b-x<\delta_{1}$, and $(b-x)^{2} \varphi(t) \leqq \delta_{2}$, the function

$$
\begin{aligned}
\tilde{G}(t, x) & =(b-x)^{2} G\left((b-x)^{2} \varphi(t), x\right) \\
& =(b-x)^{4} \int_{0}^{t} F\left((b-x)^{2} \varphi(t), x\right) \varphi^{\prime}(t) d t
\end{aligned}
$$

is a convex function of $t$,

(2) there exists a nondecreasing function $h(x)$, defined for $0 \leqq b-x<\delta_{1}$ and such that for $0<(b-x)^{2} t \leqq \delta_{2}$

$$
\begin{aligned}
& \log \left[\left(b-x_{2}\right)^{4} F\left(\left(b-x_{2}\right)^{2} t, x_{2}\right)\right]-\log \left[\left(b-x_{1}\right)^{4} F\left(\left(b-x_{1}\right)^{2} t, x_{1}\right)\right] \\
& \leqq h\left(x_{2}\right)-h\left(x_{1}\right), \text { for } x_{2} \geqq x_{1} .
\end{aligned}
$$

Then if $y$ is a solution of (1) satisfying

$$
y(b)=y^{\prime}(b)=0
$$

then $y(x) \equiv 0$ for $0<b-x<\delta_{1}$.

REMARK. When $y F\left(y^{2}, x\right)$ is nondecreasing in $y$ for $y>0$, which is the case for equation (2), then the function $\varphi$ in the hypothesis of Theorem A.7 can be taken to be $t^{2}$. On the other hand, when $y F\left(y^{2}, x\right)=q(x) f(y)$ and $y f(y)>0$ whenever $y \neq 0$, which is the case for many of the more important examples, then the function $\varphi$ may be taken as $\varphi(t)=\Sigma^{-1}(t)$ where $\Sigma(t)=2 \int_{0}^{t} f(u) d u$.

Proof of Theorem A.7. We consider the case where $F$ is of class $C^{1}$, and define a function $\Gamma(\omega, x)$ by

$$
\Gamma(\widetilde{G}(t, x), x)=t
$$

so that

$$
\begin{gathered}
\Gamma_{\omega}(\omega, x)=\left[\tilde{G}_{t}(\Gamma(\omega, x), x)\right]^{-1}, \\
\Gamma_{x}(\omega, x)=-\left[\widetilde{G}_{x}(\Gamma(\omega, x), x) / \widetilde{G}_{t}(\Gamma(\omega, x), x)\right] .
\end{gathered}
$$

Let $y$ be a solution of (1) satisfying (31) and let $\Phi(x)$ be defined by formula (13). Then, as shown in the proof of Theorem A.1,

$$
\begin{aligned}
\Phi^{\prime}(x) & =\frac{\partial}{\partial x}\left[(b-x)^{2} G\left(\alpha^{2}(b-x)^{2}, x\right)\right]_{\alpha=y /(b-x)} \\
& =\left.(\partial / \partial x) \widetilde{G}(t, x)\right|_{t=\varphi^{-1}\left((y,(b-x))^{2}\right) .}
\end{aligned}
$$

If we put $\psi(x)=\Gamma(\Phi(x), x)$, then by (33), (34) and (35)

$$
\psi^{\prime}(x)=-\left[\left.\tilde{G}_{x}(t, x)\right|_{\left.\varphi-1_{\left((y /(b-x))^{2}\right.}\right)} ^{\psi(x)}\right] \tilde{G}_{t}(\Gamma(\omega, x), x) .
$$

We observe that $\Phi(x) \geqq \widetilde{G}\left(\varphi^{-1}\left((y /(b-x))^{2}\right), x\right)$ so that

$$
\psi(x)=\Gamma(\Phi(x), x) \geqq \varphi^{-1}\left((y /(b-x))^{2}\right) .
$$


By (36) and (29)

$$
\begin{aligned}
\psi^{\prime}(x)=- & {\left[(b-x)^{4} F\left((b-x)^{2} \varphi(\psi(x)), x\right) \varphi^{\prime}(\psi(x))\right]^{-1} } \\
& \cdot \int_{\varphi-1\left((y /(b-x))^{2}\right)}^{\psi(x)} \frac{\partial}{\partial x}\left[(b-x)^{4} F\left((b-x)^{2} \varphi(s), x\right)\right] \varphi^{\prime}(s) d s,
\end{aligned}
$$

so by the convexity of $\tilde{G}$,

$$
\psi^{\prime}(x) \geqq-\int_{\varphi^{-1}\left((y /(b-x))^{2}\right)} \frac{\left\{(\partial / \partial x)\left[(b-x)^{4} F\left((b-x)^{2} \varphi(s), x\right)\right]\right\}_{+} \varphi^{\prime}(s) d s}{(b-x)^{4} F\left((b-x)^{2} \varphi(s), x\right) \varphi^{\prime}(s)} .
$$

The inequality (30) implies that the integrand in the above integral is bounded above by $h^{\prime}(x)$. Thus we obtain $\psi^{\prime}(x) \geqq-h^{\prime}(x) \psi(x)$, and integration of this inequality yields

$$
\psi(b) \geqq \psi\left(x_{0}\right) \exp \left(h\left(x_{0}\right)-h(b)\right),
$$

for $0 \leqq b-x_{0}<\delta_{1}$. Suppose now that $y \equiv 0$ for $0 \leqq b-x \leqq \delta_{1}$, then by Lemma A.2, $y$, and hence $y^{\prime}$, has infinitely many zeros, clustering at $b$ (they cannot cluster on the left of $b$ ). But at a zero of $y^{\prime}$

$$
\psi(x)=\Gamma(\Phi(x), x)=\Gamma\left(\tilde{G}\left(\varphi^{-1}\left((y /(b-x))^{2}\right), x\right), x\right)=\varphi^{-1}\left((y /(b-x))^{2}\right),
$$

and thus (37) implies that $\lim _{\sup _{x \rightarrow b}}(y /(b-x))^{2}>0$, which contradicts (31), therefore $y \equiv 0$ in $0<b-x<\delta_{1}$. In the case where $F$ is not of class $C$, we may approximate $F$ by a sequence of $C^{1}$ functions in the same manner as in the proof of Theorem A.1.

Restricting Theorems A.6 and A.7 to the special equation (2) with $0<\gamma<1$, we obtain

COROLlaRY A.5. Let $q(x)$ be positive on $[a, b]$. If $\log q(x)$ has finite lower variation on $[a, b]$, then for $x_{0} \in[a, b]$, every solution of (2), with $0<\gamma \leqq 1$, satisfying (27) vanishes identically in $\left[a, x_{0}\right]$.

Corollary A.6. Let $q(x)$ be positive on $[a, b]$. If $\log (b-x)^{\gamma+3} q(x)$ has finite upper variation as $x \rightarrow b$, and $y(x)$ is a solution of $(2)$, with $0<\gamma \leqq 1$, satisfying $y(b)$ $=y^{\prime}(b)=0$, then there exists $\varepsilon>0$ such that $y(x) \equiv 0$ for $x \in[b-\varepsilon, b]$.

Finally, we wish to make a few comments concerning the method of proof discussed here in connection with the continuability problem for superlinear equations and the uniqueness of the zero solution for the sublinear equations. In the former case, we devise certain techniques involving Lyapunov-like functions to obtain a contradiction to Lemma A.1 by either showing that (i) $\lim \sup _{x \rightarrow b}\left|y^{\prime}(x)\right|$ is finite or (ii) lim $\sup _{x \rightarrow b}|y(x)|$ is finite. In Ullrich's result [A-9], quoted above, condition (8) is used to show that (i) is true. Theorem A.2 follows the alternate route, namely by showing that the assumed conditions imply (ii). Similarly, in case of uniqueness of zero solution, one devises "dual" techniques involving 
Lyapunov-like functions to obtain a contradiction to Lemma A.2 by either showing that (i) $\lim \sup _{x \rightarrow b}\left|y^{\prime}(x)\right|>0$ or that (ii) $\lim \sup _{x \rightarrow b}|y(x)|>0$. Thus, in Theorem A. 6 we show under the given assumptions that the contrary of the desired conclusion leads to (i). Alternately, we employ the route (ii) in Theorem A.7.

\section{APPENDIX REFERENCES}

[A-1] C. V. Coffman and D. F. Ullrich, On the continuation of solutions of a certain nonlinear differential equation, Monatsh. Math. 71 (1967), 385-392. MR 37 \#3078.

[A-2] C. V. Coffman, On the positive solutions of boundary-value problems for a class of nonlinear differential equations, J. Differential Equations 3 (1967), 92-111. MR 34 \#4593.

[A-3] S. P. Hastings, Boundary value problems in one differential equation with a discontinuity, J. Differential Equations 1 (1965), 346-369. MR 31 \#4954.

[A-4] P. Hartman, Ordinary differential equations, Wiley, New York, 1964. MR 30 \#1270.

[A-5] J. W. Heidel, Uniqueness, continuation, and nonoscillation for a second order nonlinear differential equation, Pacific J. Math. (to appear).

[A-6] D. V. Izjumova and I. T. Kiguradze, Some remarks on the solutions of the equation $u^{\prime \prime}+a(t) f(u)=0$, Differencial'nye Uravnenija 4 (1968), 589-605. (Russian) MR 37 \#3128.

[A-7] R. M. Moroney, Note on a theorem of Nehari, Proc. Amer. Math. Soc. 13 (1962), 407-410. MR 26 \#6479.

[A-8] R. A. Moore and Z. Nehari, Nonoscillation theorems for a class of nonlinear differential equations, Trans. Amer. Math. Soc. 93 (1959), 30-52. MR 22 \#2755.

[A-9] D. F. Ullrich, Boundary value problems for a class of nonlinear second-order differential equations, J. Math. Anal. Appl. 28 (1969), 188-210. MR 39 \#7203.

[A-10] D. Willett and J. S. W. Wong, Some properties of the solutions of $\left[p(t) x^{\prime}\right]^{\prime}+q(t) f(x)=0$, J. Math. Anal. Appl. 23 (1968), 15-24. MR 37 \#1707.

Department of Mathematics, Carnegie-Mellon University, Pittsburgh, PennSYLVANIA 15213

Current address (Wong): Department of Mathematics, University of Iowa, Iowa City, Iowa 52240 